1 Running head: Everyday-life and experimental habits

2

3

4

5

6

7

8

9

10

11

12

13

14

15

\title{
Striatal role in everyday-life and laboratory-developed habits
}

\author{
Pasqualina Guida ${ }^{1,2,6 *}$, Mario Michiels ${ }^{1,2,6 *}$, Peter Redgrave ${ }^{5}$, David Luque ${ }^{3,4}$, Ignacio
}

Obeso $^{1,2}$

* Co-first authors

${ }^{1}$ CINAC, Hospital Universitario HM Puerta del Sur, Móstoles, Madrid, Spain

${ }^{2}$ CIBERNED, Instituto de Salud Carlos III, Madrid, Spain

${ }^{3}$ Departamento de Psicología Básica, Autonoma de Madrid University, Madrid, Spain

${ }^{4}$ Departamento de Psicología Básica, Universidad de Málaga, Madrid, Spain

${ }^{5}$ Department of Psychology, University of Sheffield, Sheffield, S10 2TN, UK

${ }^{6} \mathrm{PhD}$ program in Neuroscience, Autonoma de Madrid University, 28029 Madrid, Spain

9 Correspondence to Dr. Ignacio Obeso

20 Avda. Carlos V, 70

21 Móstoles, 28938

22 Spain

3iobeso.hmcinac@hmhospitales.com 
Everyday life \& Experimental Habits

\section{Abstract}

The dorsolateral striatum plays a major role in stimulus-response habits that are learned in the experimental laboratory. Here, we use meta-analytic procedures to identify the neural circuits activated during the execution of stimulus-response behaviours acquired in everyday-life and those activated by habits acquired in the laboratory. In the case of everyday-life habits we dissociated motor and associative components. We found that motor-dominant stimulus-response associations developed outside the laboratory engaged posterior dorsal putamen, supplementary motor area (SMA) and cerebellum. Associative components were also represented in the posterior putamen. Meanwhile, newly learned habits relied more on the anterior putamen with activation expanding to caudate and nucleus accumbens. Importantly, common neural representations for both naturalistic and laboratory based habits were found in posterior left and anterior right putamen. Our findings suggest a common striatal substrate for behaviours with significant stimulus-response associations, independently of whether they were acquired in the laboratory or everyday-life. 
Everyday life \& Experimental Habits

\section{Introduction}

Much of human behaviour can become automated and executed without conscious thought and attention. Thus, over time, a vast array of sometimes sophisticated movements, thoughts, attitudes and motivations can be performed under automatic stimulus-response control. Typically, we refer to such behaviour as habits. Throughout life, repeated associations between representations of specific stimuli and particular responses enable stimulus-evoked behaviour to be enacted without conscious intervention ${ }^{1}$. This allows us to perform tasks with reliable stimulus-response components without thought, while at the same time, allowing conscious attention to be directed to less predictable aspects of the world. An obvious example would be not having to think about putting one foot in front of the other when walking to an interview. Hence, numerous habitual routines are constructed by humans to manage the simultaneous enactment of multiple tasks - walking and talking ${ }^{2}$.

A significant feature of habitual behaviour is it occurs independently of how valuable or appropriate the outcome is ${ }^{3,4}$. An example would be habitually pressing the lift/elevator button taking you to the floor of your old office, rather than the new one. Automatic habitual control is often contrasted with conscious goal-directed processing where action selection is determined by the value/appropriateness of the predicted outcome ${ }^{3,5,6}$. Typically, before statistical regularities in the task are established, adaptive goal-directed control is slower but more flexible. Thus, early in instrumental learning, and in uncertain situations, flexible goaldirected control is normally deployed.

Until recently, habits have been studied in experimental settings where new stimulusresponse relationships are learned in the laboratory. However, it is of great interest to understand how automatic habitual control operates in the normal circumstances of everyday life. It is also important to understand how brain disorders such as Parkinson's disease ${ }^{7,8}$, obsessive-compulsive disorder ${ }^{9}$, and drug addictions ${ }^{10}$ lead to clinically dysfunctional patterns of habitual use of behaviour. Despite the importance of habitual 
Everyday life \& Experimental Habits

control in daily life and clinical pathology, formal investigation of the inherent stimulusresponse associations established in everyday life is in its infancy. However, even at this early stage, an important question is whether the neural circuits engaged by habits acquired in daily life are the same or different to those activated when newly acquired habits are performed in the laboratory. The purpose of the present investigation was therefore to compare the patterns of neural activation evoked by long-acquired habits brought into the laboratory with those established under formal experimental conditions.

Experimental research with animals has shown how instrumental learning occurs through initial goal-directed computations that later transition into stimulus-response mappings ${ }^{11}$. The formal experimental paradigms (see Box 1 for a summary) used to distinguish habits from goal-directed actions include (amongst others) outcome devaluation or contingency degradation tests (Adams, 1982; Balleine and O'Doherty, 2010; Dickinson et al., 1985; for a review see Foerde, 2018). In both cases, if the behaviour persists after the outcome has been devalued or the outcome is no longer related to responding, the behaviour is deemed to be under habitual control. The formal procedures developed in animal studies have been imported into human experiments ${ }^{14-18}$. Significantly, human neuroimaging studies have revealed activation of the rostro-medial (associative) striatum during the initial stages of instrumental learning, which gradually shifted to caudo-lateral (sensorimotor) regions of the striatum when habitual control of the instrumental task became evident ${ }^{14,19-24}$. These findings concur with those in non-human animals that demonstrate a similar involvement of the rostro-medial striatal territories early, and caudo-lateral regions later in the acquisition of instrumental tasks ${ }^{11}$.

To date, most of the literature investigating the neural basis of habits in humans has relied on subjects developing new experimental habits in the laboratory under formal experimental conditions (Box 1B). This approach, has provided important information about the relationship between habitual and goal-directed neural systems ${ }^{11}$ and the factors 
Everyday life \& Experimental Habits

95

96

(repetition, rewards or environmental cues) that promote the formation of habitual control ${ }^{25}$. However, this approach faces several challenges. The most pressing is the difficulty of creating habits in an experimental situation that are equivalent to those developed in normal everyday life. For example, a basic tenet of habit theory is that stimulus-response associations typically get stronger with repetition. It is therefore, not exactly inspiring that several studies have failed to report a direct positive relationship between the amount of training and the strength of habitual responses measured in their experimental settings ${ }^{26,27}$. have everyday habitual behaviour learned during a subjects lifetime brought into the laboratory for investigation. Certain behaviours in normal life including driving, eating, dancing, reading, talking or walking have significant stimulus-response components that can be performed automatically while the person's conscious attention is directed elsewhere ${ }^{28,29}$. It is likely these components have been acquired through a life-time of everyday trials. Therefore, such associations come to the laboratory fully formed and do not depend upon on the multiple trial learning that is required to develop new experimental habitual behaviour. The principal challenge in studying naturalistic habits is getting subjects to express longestablished stimulus-response behaviour in a laboratory setting. This is necessary so that both the automatic behaviour and associated neural activity can be measured quantitatively. Investigators of everyday habits have chosen behaviours that have critical automatic stimulus-response components ${ }^{30}$ and versions that can be performed in the laboratory while BOLD signals are measured by fMRI imaging. Examples of such tasks include reading, where comparisons are made between real words of different familiarity and emotional content, foreign words and pseudo-words ${ }^{31}$; writing and drawing ${ }^{32,33}$ walking on a special apparatus ${ }^{34}$; and driving an MR-compatible driving simulator (Box 1A; Choi et al., 2017; Cummine et al., 2016; Huth et al., 2016; Karimpoor et al., 2015; Martínez et al., 2016, 2018; Oberhuber et al., 2013; Varotto et al., 2020; Yang et al., 2018). The specific question we 
Everyday life \& Experimental Habits

121

122

were interested in is whether recently established laboratory habits and those developed in everyday life engaged the same, partially overlapping or separate neural circuits in the brain.

The purpose of the present investigation was therefore to directly compare the neural signatures evoked by novel, laboratory-developed habits and those acquired over a life-time (Figure 1). To answer this question, we conducted a quantitative meta-analysis to investigate the neural substrates of everyday life and experimental habits. First, to select a cohort of studies investigating naturalistic habits, we took data from 54 studies (a total of 1441 subjects) that had used diverse stimulus-response paradigms (walking, reading, writing and driving). Imaging models that included automatic parameters on each task were chosen (Table S1). Meanwhile, we included categorical variables that separated motor (walking, driving) and cognitive (reading, writing) habits acquired in daily life, a further sub-division motivated by the anatomo-functional gradient along fronto-striatal circuits ${ }^{41}$. Second, we sought to confirm the neural basis of experimental habits in the 40 studies (a total of 973 subjects) that had used probabilistic or discriminative learning, 2-step learning or sequential tasks to test for the laboratory development of novel habits (Box 1B). Studies of experimental habits were separated into two subcategories: probabilistic learning vs other experimental tasks (Table S2), on the ground that probabilistic classification typically activates more anterior portions of the caudate and the putamen, compared with other tasks 22. This novel approach allowed the neural signatures of stimulus-response behaviour developed in the laboratory and in everyday life to be compared directly. Special interest was focused on the system-level circuits involving the basal ganglia (Figure 1). The resulting information provides important clues into the organisation of normal habits against which pathologies of habit and the results of therapeutic interventions can be referenced. 
Everyday life \& Experimental Habits

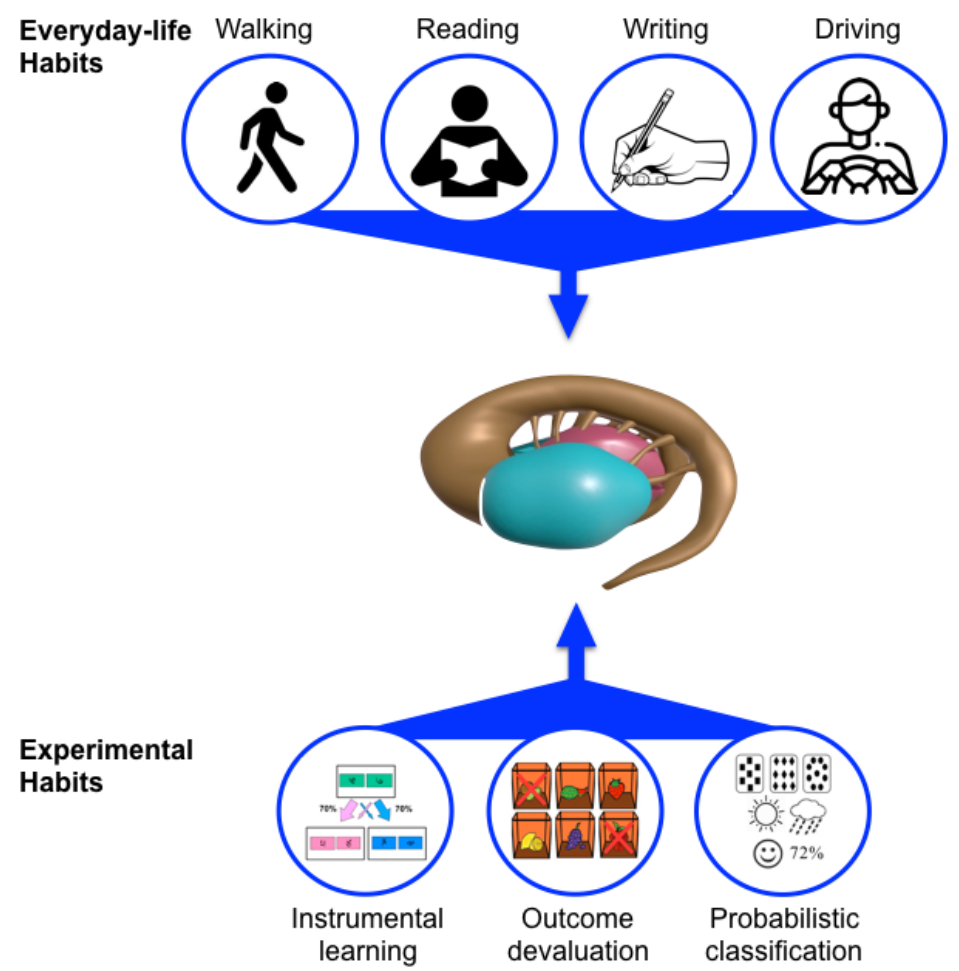

Figure 1. Hypothesis diagram on striatal role in both everyday-life and experimental habits. Activities part of daily life such as writing, reading, walking or driving were selected as everyday-life habits (see Box 1 for task measurements and details) to expect a critical striatal role in executing these habits forms. Similar striatal activities can be expected compared to experimental paradigms commonly used in the cognitive science literature.

\section{Methods}

Selection of studies. We conducted 2 independent searches in PubMed to identify fMRI studies investigating stimulus-response habits acquired (1) in everyday life and (2) in experimental laboratories. The search of articles related to everyday habits was focused on natural behaviours where an important automatic stimulus-response component would be expected (e.g. walking, speech, reading, etc). Selection of these activities was based on their potential link to fundamental basal ganglia functions (Figure 1). A summary of typical measures used in these studies is presented in Box 1. In the search for laboratory based habits we included articles referenced by Patterson \& Knowlton (2018) plus all new relevant articles published since their last search (June 22, 2017) and March 26, 2021. To 
Everyday life \& Experimental Habits

enable direct comparison with, and extension of, the Patterson and Knowlton review (2018), we conducted our literature search using the same key search terms and included the task categories - probabilistic learning, discriminative learning and sequence learning (see Box 1). We also included relevant MeSH terms available in Pubmed. This allowed us to find articles that do not include targeted keywords in the title or abstract but have proper MeSH terms linked to their metadata. The complete list of our search terms can be found in Supplementary Material. To set limits on inclusion, the articles were filtered according to the following criteria:

(1) Spatial coordinates from human brain fMRI reported in standardized stereotaxic space (MNI or Talairach space). Other functional imaging methods (e.g., PET, EEG source imaging, etc) were excluded;

(2) Healthy subjects over 18 years old were included, as were the healthy participants within clinical studies with independent analyses for the healthy controls;

(3) At least 6 subjects;

(4) Whole-brain analysis. ROI analyses were excluded as they can be biased by the study hypothesis and may not report all significant regions;

(5) For studies using the two-step probabilistic tasks and analyzing prediction errors from computational models, we included results associated with model-free (reward prediction error, RPE), but not from model-based errors (state prediction error, SPE);

(6) When a study contained multiple experiments and/or contrasts, the contrasts selected were based on the task condition and comparisons linked to stimulus-response behaviours (see Table S1 and S2);

(7) Studies reporting original data and published in English peer-reviewed journals (reviews and meta-analysis were excluded).

We then performed a second-step control using reference lists from already included articles. From the list of references, we first excluded articles whose title had no direct link to any of our inclusion criteria. From the remaining articles that met our inclusion 
Everyday life \& Experimental Habits

criteria we found 17 that had investigated everyday-life habits and 14 articles that had investigated laboratory-developed habits. Since our second exclusion step involved manual, rather than online searching, we were able to include articles published before 2017 not included in Patterson and Knowlton (2018). These additional references were selected because: (1) we checked all references from the list of accepted papers, not only those in major review articles; and (2) our criteria allowed us to include studies that did not report putative habit-related activation of the dorsal striatum (caudate, putamen, or both). Figure S1 depicts the identification, screening, eligibility and selection stages we used to select studies for inclusion.

We included a total of 54 studies investigating everyday-life habits (31 involved cognitive tasks: writing, reading; and 23 studied motor tasks: walking, driving; Table S1). A total of 40 studies involved reported experimental habits (20 involved probabilistic learning; and 20 studied other experimental tasks; Table S2). Foci, scripts and statistical maps can be accessed in the Open Science Framework (https://osf.io/w5ftm).

Data analysis. The latest version of the GingerALE software v3.0.2 ${ }^{42}$ was used to compute activation likelihood estimations (ALE) ${ }^{43}$. From each of the accepted articles, the coordinates of peak activations were manually extracted and those in Talairach space were transformed to Montreal Neurological Institute $(\mathrm{MNI})$ space using the inverse transform of icbm2tal from GingerALE ${ }^{44}$. The ALE method was implemented as follows: (1) for each study a 3D Gaussian distribution was created around every peak activation (variance proportional to the sample size of the study). This allowed us to use the higher statistical power to reduce peak uncertainty in studies with larger sample sizes. This step was done for every peak to produce one modelled activation (MA) map per study. The value for each voxel in a MA-map represented the probability of that voxel containing an activation foci. (2) Voxel probabilities in all MA-maps were merged to produce an ALE map. Following recommendations of Eickhoff et al., (2016), the ALE map was thresholded using a cluster- 
Everyday life \& Experimental Habits

214

level Family-Wise Error (FWE), with a cluster-forming threshold of .001 and a cluster-level FWE of .05 (as stated in the GingerALE manual; Fox et al., 2013).

The first step in applying a cluster-level FWE was to threshold the ALE map at the voxel-level (cluster-forming threshold). To do this, all possible combinations between all peaks from each MA-map were tested. The ALE values provided a null distribution which assumed the peaks would be randomly distributed following all potential combinations amongst them. Here, an uncorrected $p$-value was used since in the next step, a FWE correction was applied to correct for the possibility of multiple comparison errors.

Then, an additional threshold (cluster-level FWE) was applied to select only the largest clusters. Peaks in every MA-map were distributed randomly and then combined into a single ALE map, following the same union method described above for the voxel-level threshold. The ALE maps were also thresholded with the same method used at the voxellevel threshold. This procedure was repeated (1000 times for this meta-analysis) using Monte Carlo permutations method, selecting on every run the largest cluster in the thresholded ALE map (null distribution). Finally, we applied the selected threshold (0.05) to obtain the FWE-corrected results. Using a 0.05 cluster-level threshold resulted in a thresholded map where only $5 \%$ of the surviving clusters could have been introduced by chance (false positives), following guidelines to discard non-significant clusters ${ }^{42}$.

Contrasts between thresholded ALE maps were computed to compare between our conditions of interest (e.g. Everyday-life > Experimental maps). All foci in the selected studies were pooled into a single dataset, which we then split in two datasets by randomly assigning to each one the same number of foci in their original file. The ALE-scores of these two random maps were then subtracted in a voxel-wise manner generating the map of their ALE-scores differences (repeated 10000 times to record ALE-scores and generate a null distribution of randomly spatially distributed foci). Finally, the actual two ALE maps corresponding to the contrast were combined voxel-wise by subtracting the ALE-score of 
Everyday life \& Experimental Habits

one map from the other. The resulting map of ALE-scores were then thresholded voxel-byvoxel by comparing them with the null distribution previously obtained. We used the default p-value suggested in GingerALE (0.01 uncorrected). All computations were run via in-house python scripts to automate analyses in the GingerALE's interface. Thresholded maps are reported in MNI152 space ${ }^{47}$.

\section{Results}

\section{Striatal role in everyday and experimental habits}

To find the neural signatures of everyday habits, we obtained cluster activity associated with stimulus-response behaviours learned throughout life, compared with those newly learnt in an experimental laboratory. The main effects of naturalistic habits revealed significant bilateral activity in the posterior putamen (Table 1; Figure 2A). Specifically, this was sustained by activity in dorsal sections and left putamen activation that expanded to its posterior boundary (Table 1; Figure 2A-B). For these tasks, other active regions were seen in the cerebellum and cortical areas including the premotor and SMA (Table 1; Figure 2A-B).

Habits established in laboratory settings were also associated with increased levels of activation in putaminal sections, but with larger representations in anterior striatum (Figure 2C). A gradient was seen along the rostro-ventral section of the putamen, right caudate and the nucleus accumbens bilaterally (Table 2; Figure $2 \mathrm{C}$ ). These results confirm the striatum as the most significant region for newly acquired experimental habits (Table 2; Figure $2 \mathrm{C})^{22}$. They were also consistent with the idea that stimulus-response associations learned in the laboratory would depend more on rostral striatal activity. The right insula was one of the extrastriatal hubs of activity associated with habits acquired in the laboratory (Table 2; Figure 2A). These findings align with the evidence that sensorimotor territories of the striatum are a critical for the expression of habitual behaviour, but with more rostral patterns of activity observed when experimental habits were developed in laboratory setting. 
Everyday life \& Experimental Habits

267 differences between neural activation patterns associated with everyday and experimental

268 habits, there may be a common neural substrate accessed by both forms of stimulusresponse behaviour. Common activations were seen in anterior right putamen and posterior left putamen (Figure 2A). Although both categories of habits recruited dorsal sub-regions of the putamen bilaterally, the activation by everyday habits was stronger (Figure 2C).

272 Unexpectedly, no activation in the caudate nucleus survived FWE-corrected thresholds with 273 everyday habits. In contrast, habits acquired in the laboratory showed a differential

274 recruitment of the nucleus accumbens, and to a lesser extent, the most antero-ventral

275 section of the right caudate nucleus and putamen (Figure $2 \mathrm{C}$ ). Hence, while the striatum is a

276 common hub for both categories of habitual behaviour, antero-posterior differences were

277 reflected in the activation patterns of habits acquired in everyday life and the experimental

278 laboratory. 
Everyday life \& Experimental Habits

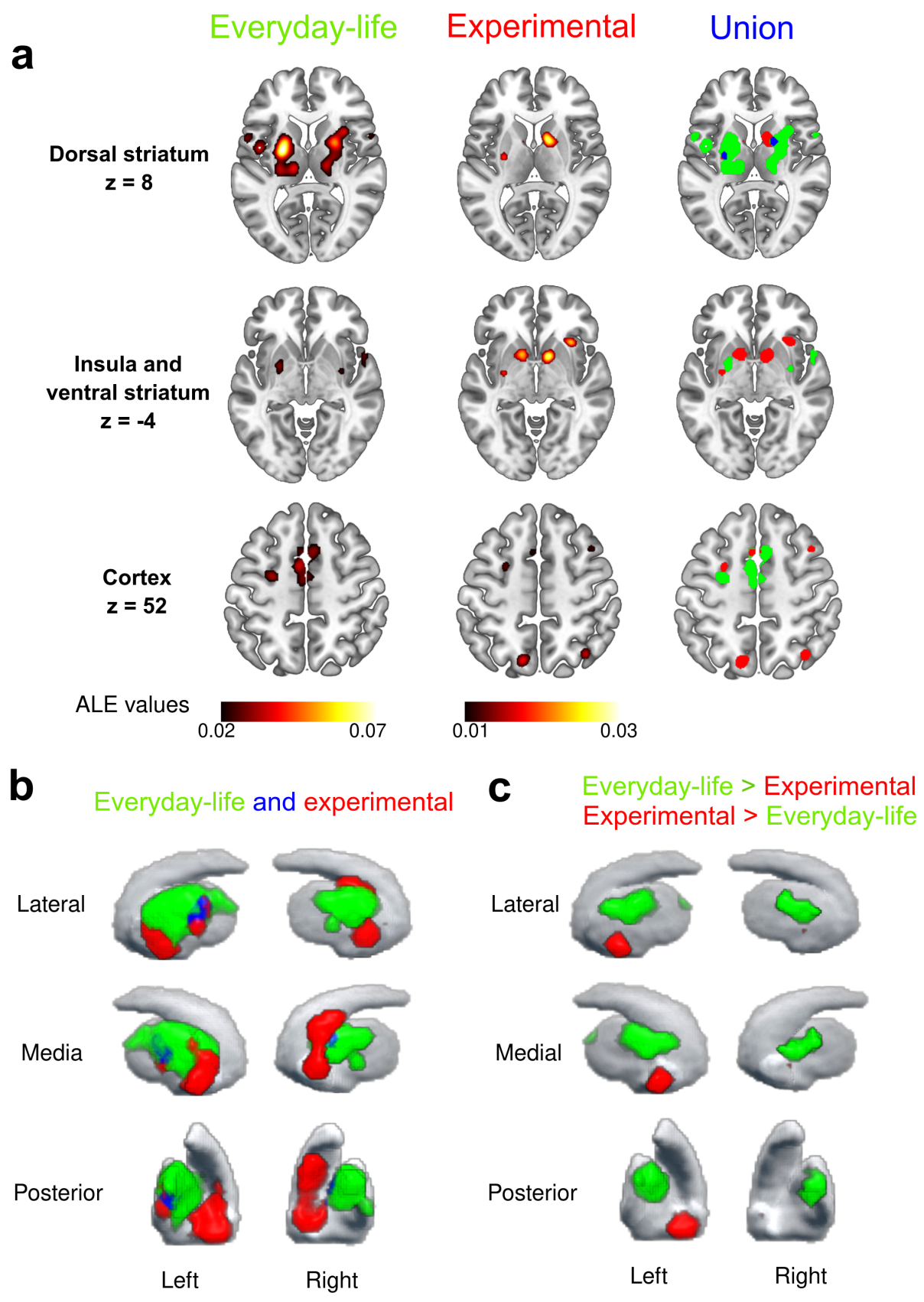

Figure 2. Everyday-life and experimental thresholded activation maps. a) Axial views for the main cerebellar cortex for the case of everyday-life studies but it is omitted here for brevity. Z=52 view for the experimental studies is shown as an unthresholded map for visualization purposes (ALE value $\approx$ 0.01 ) (b) $3 D$ striatum reconstruction showing all the activation that fall inside it. Overlap regions at the right column are shown in blue. c) 3D striatum reconstruction showing the differential activation of the Everyday -life > Experimental contrast (in green) and the Experimental > Everyday-life contrast (in red). 
Everyday life \& Experimental Habits

We next tested whether the neural correlates of everyday habits varied between sensorimotor routines (walking or driving) and cognitive-associative ones (reading or writing). While all representations of habitual behaviour are characterized by stimulusresponse associations, for different tasks these may be represented in different sensorimotor networks with differential connections to the basal ganglia. Clustered activation in motor tasks revealed a significant presence along the dorsal putamen compared with the associative tasks (Figure 3A). In addition, motor tasks extended the pattern of activation into more posterior sections of the right putamen, but only dorsally for the left putamen. This cluster was present across all the antero-posterior axes (Figure 3B).

In the case of associative habits acquired in everyday life, we observed an activation pattern in ventral sections of the putamen bilaterally (Figure $3 A$ ). This effect showed significant bilateral asymmetry favouring the left putamen where activation extended into more posterior regions. This was absent in the walking and driving tasks (Figure 3A).

Outside the striatum at the cortical level, the associative tasks acquired in everyday life recruited premotor area and SMA. Activation was also observed in the cerebellum (Figure 3A). The same cortical regions were recruited during the walking and driving tasks, but none survived FWE-corrected thresholds. Greater activation for the associative cognitive tasks was expected given the more complex computational requirements when writing or reading ${ }^{48}$. Such tasks are known to engage larger regions of cortical tissue ${ }^{49}$.

To further investigate the failure of our thresholding procedures to detect activation in the caudate nucleus for any of the naturalistic habit categories, we included the distribution of all the foci that fell into the striatum in both categories of everyday habits (Figure $3 \mathrm{C}$ ). This allowed activity in the right caudate to be observed (Figure $3 C$ ) in some associative studies, but not for the motor ones. For the left caudate nucleus, no foci were reported in any study, which confirms the absence of activity reported in the thresholded maps above. 
Everyday life \& Experimental Habits

These findings provide insights into possible parallel habitual mechanisms in motor-

316 associative domains that may share regions of the posterior putamen for motor activities, but

317 a broader network for actions that require higher-order cognition. Given the stimulus-

318 response associations involved in everyday habits will simultaneously engage multiple

319 mechanisms, including visuo-spatial attention, movement planning and execution, the neural

320 substrate of cognitive and sensorimotor habits should be segregated. 
Everyday life \& Experimental Habits

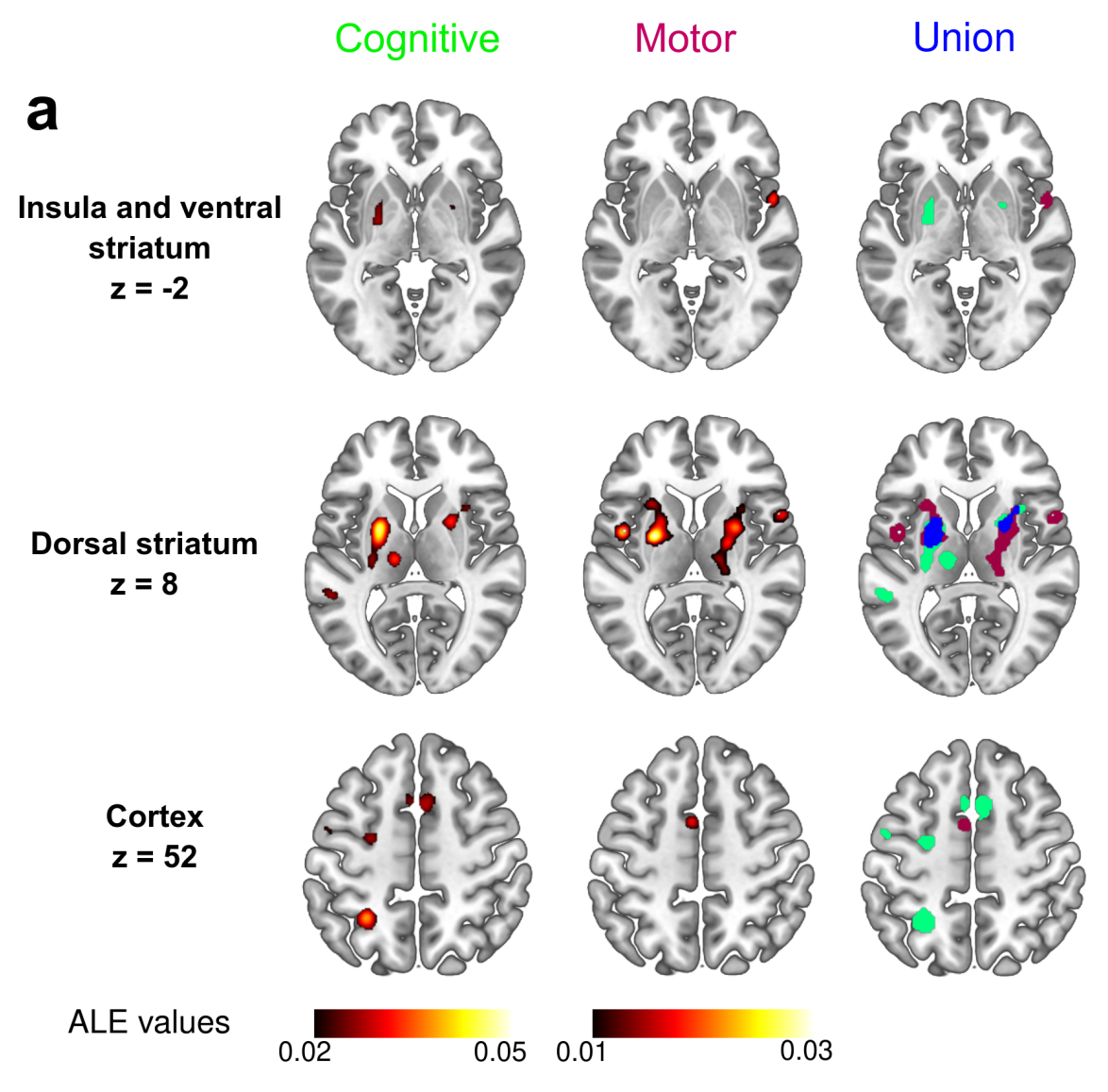

\section{b}

Lateral
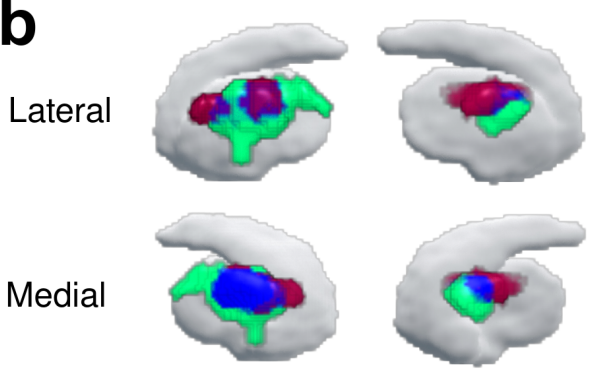

Posterior

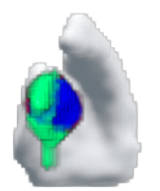

Left

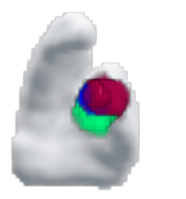

Right
C

Lateral
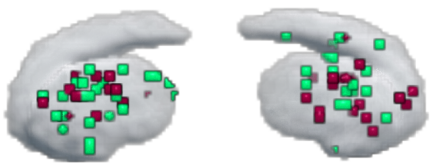

Medial
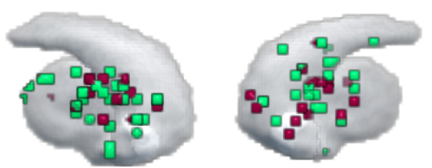

Posterior

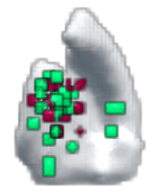

Left

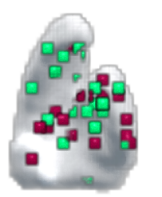

Right

Figure 3. Everyday-life cognitive-motor subcategories thresholded activation maps. a) Axial views for the main regions. Overlap regions at the right column are shown in blue. b) $3 D$ striatum reconstruction showing all the activation that fall inside it. Overlap regions at the right column are shown in blue. c) Foci distribution of all the studies that fall in the striatum. Note that some foci may correspond to the same study. 
Everyday life \& Experimental Habits

\section{Probabilistic learning and other tasks segregation in experimental habits}

Finally, we aimed to replicate previous findings on laboratory conditions that have linked the striatum to the learning and execution of new habits. Consistent striatal activity has been reported when evaluating probabilistic or discriminative learning, 2-step learning and sequential tasks ${ }^{22}$. Here, we intended to confirm and update these results with more recent findings by separating studies that involve trial-and-error probabilistic reward learning from those that used different methodologies for stimulus learning. As predicted, both probabilistic learning and the other tasks showed common regions of striatal activation, with largest clusters in the nucleus accumbens and rostro-ventral sections of the caudate and putamen (Figure 4A). However, only the probabilistic tasks were associated with bilateral recruitment of the most anterior region of the putamen (Figure 4B). This results confirms previous findings reported by Patterson and Knowlton, (2018). Similarly, the other experimental tasks differed with respect to the probabilistic ones by demonstrating unilateral recruitment of the left rostral caudate nucleus (Figure 4B). Outside the striatum, left insular cortex was activated in other tasks but not by the probabilistic ones (Figure 4A). This could reflect a form of residual goal-directed activity due to the insula's complementary role in evaluating future rewards in the decision phase ${ }^{50}$. 
Everyday life \& Experimental Habits

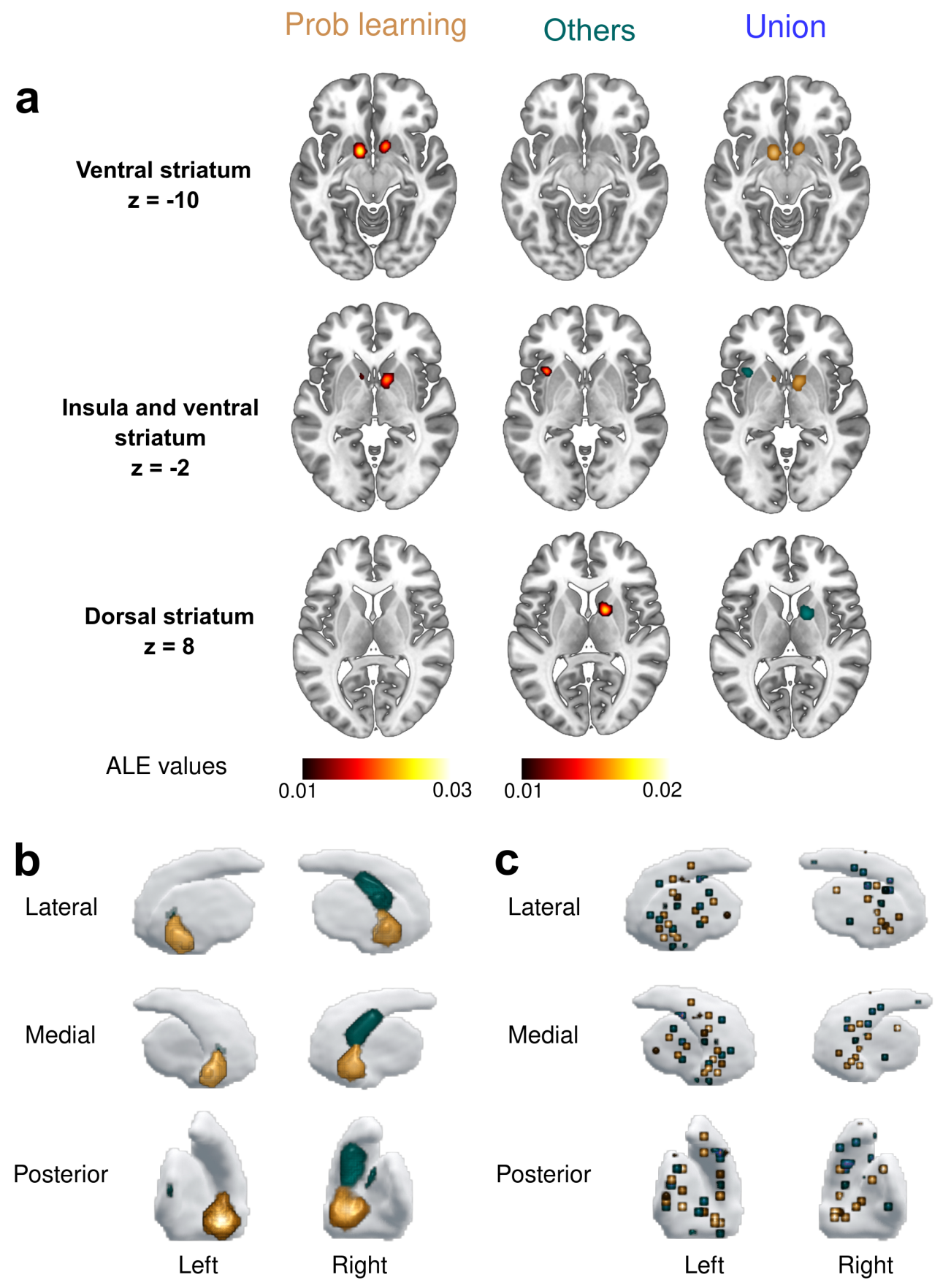

Figure 4. Experimental subcategories thresholded activation maps. a) Axial views for the main regions. In this case there are no overlap regions at the right column. b) $3 D$ striatum reconstruction showing all the activation that fall inside it. In this case there are no overlap regions at the right column. c) Foci distribution of all the studies that fall in the striatum. Note that some foci may correspond to the same study. 
Everyday life \& Experimental Habits human striatum, by comparing long-established habits acquired in everyday life with newly learned habits acquired under laboratory-controlled conditions. The results of our investigation revealed both common and diverse functional links between different subregions of the brain's habitual circuitry. Naturalistic habits showed enhanced activity in the dorsal posterior putamen, together with activity in the cerebellum and SMA. In contrast, laboratory acquired habits engaged anterior sections of the putamen with activation expanding to caudate nucleus and nucleus accumbens. However, common regions of activation were found in posterior left and anterior right putamen. Ultimately, delineation of specific striatal contributions to motor-associative variables embedded in habits were responsible for shared anatomical patterns in everyday-life associative habits, such as reading and writing, that engaged rostral regions of the putamen. in studies of long-established habits compared with newly acquired experimental ones. This may implicate a broad putaminal role for the stimuli-rich sensorimotor computations required during complex stimulus-response tasks enacted in everyday life. Consistent with this view, neuroimaging, lesion and animal electrophysiological data all converge to pinpoint a role for the putamen in the integration of movement units and stimulus associations to produce behaviour with a predicted outcome ${ }^{51-55}$. Indeed, the neurophysiological properties of the putamen are supported by a subpopulation of neurons that respond to sensory stimuli ${ }^{56}$, unifying actions sets for movement sequences ${ }^{57,58}$ or integrating elementary movement units such as individual finger moves ${ }^{59}$. Moreover, its activity is not solely dedicated to movement parameters, but also in the absence of motor plans ${ }^{60}$, increasing response magnitude to the reinforced choice ${ }^{61}$ and for predicted well-learned and contextually-driven actions ${ }^{62-67}$. Hence, the diversity of sensory-related and high-order reinforcement neurons in the putamen support a pivotal role in context-rich scenarios at several levels (sensorimotor, 
Everyday life \& Experimental Habits

predicted actions, object value, habitual action execution). We interpret the association of putamen activity with habits in everyday life being due to its physiological and reinforcement properties contributing to action sequence-specific and context-specific behaviour.

The role of the putamen in habitual behaviour is directly influenced by ascending dopaminergic system acting on cortical and thalamic inputs ${ }^{68}$. Dopamine provides critical modulatory influences on striatal subregions whereby projections from substantia nigra pars compacta and the ventral tegmental area differentially target terminals in dorsal and ventral striatum respectively ${ }^{69}$. The main nigrostriatal projection is topographically organised with a medial to lateral gradient ${ }^{70}$. Tonic firing within this system sustains, motivational, cognitive and action-specific decision making ${ }^{71,72}$, while sensory-evoked phasic patterns of dopaminergic activity provide a general mechanism for reinforcement learning ${ }^{73,74}$. The large bilateral activity found in the striatum for the routines of everyday life should be influenced by ascending dopaminergic fibers with the abovementioned functional properties that likely contribute to sensorimotor control while writing or walking (proprioceptive and muscle control). As well, dopaminergic projections will fire phasically to unpredicted associative cues present in everyday life ${ }^{75}$. Hence, the putamen will likely respond preferentially to the sensorimotor contingences present in highly-organised actions that rely on automatic stimulus-response associations that characterise habitual responding.

A second key contributor comes from particular cortical structures that regulate the expression of habits ${ }^{23,67,76,77}$. In the present study we found that SMA activity was an essential component of the habitual circuitry present for the everyday tasks. The SMA represents one of the important junctions between cortical-subcortical motor and cognitive circuits $^{78}$. It has projections to the dorso-lateral striatum and posterior putamen ${ }^{79}$, a pathway subject to neuromodulation by non-invasive brain stimulation ${ }^{80}$. The SMA has been shown to be involved in learning stimulus-response contingencies ${ }^{78,81}$ and model-free tasks ${ }^{82}$. Multi-dimensional components required while driving or even walking (somatosensory, visuo-spatial, prediction, motor planning, etc) will be mediated via cortical 
Everyday life \& Experimental Habits

407 inputs to the putamen ${ }^{83,84}$. How the putamen might operate on this range of input would be

408 to perform sequential selections of stimuli that trigger previously acquired stimulus-response associations. When bolted together such serial selections can be viewed as coherent sequences of habitual behaviour, such as changing gear while driving, or pen movements associated with particular letters when writing. These automatic moves are likely to recruit medial cortical motor areas including the SMA, that collaborate with subcortical structures, including putamen (Cunnington et al., 2002; Smittenaar et al., 2013). Recurrent corticostriatal loops would therefore combine to mediate sequential cognitive and motor components of habitual behaviour, both triggered by the specific sensory events they have been repeatedly associated within everyday life.

To investigate the possible commonalities and differences in the neural networks supporting categories of automatic behaviours acquired in everyday life, we subdivided those activities with greater motor component (walking, driving) compared to those with stronger cognitive requirements (reading and writing). This analysis was motivated by possible differences in the contribution from motor, cognitive and/or emotional circuits in the different types of everyday habitual activity ${ }^{88}$. The dorso-lateral caudal putamen was active for both cognitive (reading, writing) and motor operations (driving, walking), which corresponds with our understanding of parallel inter-related cortico-striatal functions ${ }^{82,89}$. The stimulus-response selection role of the putamen may well represent the low level sensorimotor selections necessary in all forms of habitual motor behaviour ${ }^{60,75}$. Parallel circuits have been suggested to integrate the different environmental signals that trigger motivational, cognitive and motor responses ${ }^{90,91}$. Sequential selections in different territories of the basal ganglia could be integrated by cortico-basal ganglia loops into coherent goaldirected behaviour ${ }^{92}$. The question that remains is whether each of the limbic, associative and sensorimotor territories ${ }^{93}$ can all operate in stimulus-response habitual mode to elicit respectively, stimulus-evoked motivations (e.g. drug cravings), stimulus-evoked cognitions (prejudices) as well as the well-known motor habits. 
Everyday life \& Experimental Habits stimulus-response habits learned in experimental laboratories (probabilistic or discriminative learning, 2-step learning or sequential tasks), which also report significant striatal activations 14,19,21-23,82,94-96. Specifically, our analyses show stimulus-responses behaviour acquired both in everyday-life and under experimental conditions showed common striatal activity in the right anterior putamen and left posterior putamen. The everyday-life tasks included long established sensorimotor responses triggered by sensory cues in the absence of new associative learning. In contrast, laboratory learned habits typically involved relatively minor motor components (finger key presses), but novel cue-response associations driven by reinforcement learning. In line with a previous meta-analysis on basal ganglia activation across multiple motor disciplines (Arsalidou et al., 2013), left lateralized putaminal activity was prominent in motor operations (such as eye movements and body motion), has a larger volume in right-handed participants (Peterson et al., 1993) and is critical in behaviours guided by stimulus-response mappings ${ }^{22,95}$. Hence, we interpret left posterior putamen activation being present in both long-established and novel habit forms reflecting a critical sensorimotor association embedded within every habitual response. associated with initial learning of stimulus-response associations in experimental settings ${ }^{21}$. Activity in key regions of the circuitry associated with goal-directed behavioural control (caudate and nucleus accumbens) were also present in laboratory studies of habits. These findings match those of several fMRI studies using various reinforcement learning tasks, which report activation of the ventro-medial prefrontal cortex, insula or anterior striatum when encoding the value of predicted reward outcome linked to new actions ${ }^{20,50,98}$. Yet, the results with learning new habits may be explained by the theory and methodological procedures often used when studying habit acquisition in the laboratory. For example, part of the problem is that some of the presumed habits established in the laboratory fail to meet the 
Everyday life \& Experimental Habits

461

462

experimental studies failed to demonstrate the expected effect of training duration on the outcome-devaluation test ${ }^{17,26,27}$. This suggests rather more trials may be needed to establish stimulus-response associations that can survive devalued outcome challenges and can be enacted automatically without thought.

Recently, alternative procedures have been developed to establish and test new habits in the experimental laboratory, including the sudden reversal of learned actions after overtraining ${ }^{18}$, overloading goal-directed top-down control while measuring execution of learned stimulus-response associations ${ }^{99}$ or biasing movement kinematics ${ }^{100}$. Interestingly, pre-existing categorical associations established in everyday life (i.e. color associations or prejudice) have a clear advantage on measuring automatic processing ${ }^{30,101}$. Further methodological options for studying long established habits acquired in everyday life include assessing expert musicians ${ }^{102}$, tennis players ${ }^{103}$ and expert shooters ${ }^{104}$. Hence, getting subjects to bring their everyday-life stimulus-response associations into the laboratory under controlled conditions is an important option for studying the neural substrates underlying habitual behaviour in humans. Although there is less control of the independent variables in such studies, by selecting subjects with different amounts of everyday life experience it is possible to relate the amount of practice with habit strength. The dependent consequences of life-long habits can be measured subsequently in the laboratory with traditional outcome devaluation, contingency degradation, or dual processing procedures-

Insofar as the caudal putamen has been identified as a critical node in the neural substrate responsible for automatic habitual behaviour, malfunctioning of this region has been associated with deficits in habitual performance. One notable instance is the differential loss of dopamine neurotransmission from this region in Parkinson's disease ${ }^{7}$ a putative pathophysiological condition linked to the cost of life-long use of habits ${ }^{105}$. The problems Parkinson's disease patients have with walking and writing ${ }^{8,106,107}$ and the new learning of experimental habits ${ }^{108,109}$, have been interpreted as an inability to express stimulusresponse habits. In contrast, other neuropsychiatric complications such as addictions exhibit 
Everyday life \& Experimental Habits

an excessive cue-dependent use of certain rewards linked to increased posterior putamen activity ${ }^{10,110}$. Hence, depending on the nature of the neurobiological disruption, the habitual system seems to be underused or overused in different clinical conditions.

Despite the clear positive results of our analysis certain limitations of meta-analytic procedures must be acknowledged. First, the ALE analysis can cause some parameters, such as voxel peaks, to be overlooked. Ideally, to explore the statistical activity maps of each study individually would be of great value. However, the fact that most studies did not have full imaging datasets would preclude this. Second, we included results from prior studies whose experimental focus was not on habitual behaviours acquired in everyday life.

\section{Specifically, in these studies behaviours were not formally identified as habitual using} outcome devaluation or contingency degradation tests. However, it should be noted that a life-time repetition of everyday trials builds strong associations that do not depend upon new stimulus learning required in experimental studies ${ }^{111,112}$. Last, our meta-analysis included tasks with significant heterogeneity and regional foci were selected from studies with varying contrasts. To overcome these issues, we selected activity maps only from studies that reported contrasts measuring automatic components of behaviour. This suggests that our dataset has enabled us to identify activity patterns that are shared by a diverse range of human behaviours that contain a significant stimulus-response element. Consequently, it is possible that the bilateral posterior putamen acts as a critical node when practiced behaviour can be performed automatically and without thought in situations of everyday life.

\section{Conclusions}

The present study points to a fundamental functional role for the posterior putamen in the expression of habits acquired in everyday life. Importantly, a critical dissociation was established between different brain regions whose contributions to motor-cognitive representations of habits have, thus far, been largely indistinguishable. Conversely, the 
Everyday life \& Experimental Habits

514 engagement of the anterior putamen is associated more with habits newly learned in

515 experimental laboratories. Careful experimental protocols must be designed to identify those

516 chunks of behaviour that are under stimulus-response control and can occur independently

517 of outcome value or response contingency. This is true both for newly acquired associations

518 in the laboratory and habits established in everyday life. Finally, the present study highlights

519 the importance and value of having subjects bring life-long habits into the laboratory to be

520 investigated and compared with recently acquired stimulus-response associations. 
Everyday life \& Experimental Habits

521

522

523

524

525

526

527

528

529

530

531

532

533

534

535

536

537

538

539

540

541

542

543

\section{References}

1. Haith, A. M. \& Krakauer, J. W. The multiple effects of practice: skill, habit and reduced cognitive load. Curr. Opin. Behav. Sci. 20, 196-201 (2018).

2. Sun, R. Desiderata for cognitive architectures. Philos. Psychol. 17, 341-373 (2004).

3. Dickinson, A., Trans, P. \& Lond, R. S. Actions and habits: the development of behavioural autonomy. Philos. Trans. R. Soc. London. B, Biol. Sci. 308, 67-78 (1985).

4. Miller, K. J., Shenhav, A. \& Ludvig, E. A. Habits without values. Psychological Review 126, 292-311 (2019).

5. Daw, N. D., Niv, Y. \& Dayan, P. Uncertainty-based competition between prefrontal and dorsolateral striatal systems for behavioral control. Nat. Neurosci. 8, 1704-11 (2005).

6. Gläscher, J., Daw, N., Dayan, P. \& O’Doherty, J. P. States versus rewards: Dissociable neural prediction error signals underlying model-based and model-free reinforcement learning. Neuron 66, 585-595 (2010).

7. Redgrave, P. et al. Goal-directed and habitual control in the basal ganglia: Implications for Parkinson's disease. Nature Reviews Neuroscience 11, 760-772 (2010).

8. Bannard, C. et al. Reduced habit-driven errors in Parkinson's Disease. Sci. Rep. 9, 18 (2019).

9. Gillan, C. M. Recent Developments in the Habit Hypothesis of OCD and Compulsive Disorders. in 1-21 (Springer, Berlin, Heidelberg, 2021). doi:10.1007/7854_2020_199

10. Sjoerds, Z. et al. Behavioral and neuroimaging evidence for overreliance on habit learning in alcohol-dependent patients. Transl. Psychiatry 3, e337-8 (2013). 
Everyday life \& Experimental Habits

544 11. Balleine, B. W. \& O'Doherty, J. P. Human and rodent homologies in action control: Corticostriatal determinants of goal-directed and habitual action. Neuropsychopharmacology 35, 48-69 (2010).

12. Adams, C. D. Variations in the Sensitivity of Instrumental Responding to Reinforcer Devaluation. Q. J. Exp. Psychol. Sect. B 34, 77-98 (1982).

13. Foerde, K. What are habits and do they depend on the striatum? A view from the study of neuropsychological populations. Curr. Opin. Behav. Sci. 20, 17-24 (2018).

14. Tricomi, E., Balleine, B. W. \& O’Doherty, J. P. A specific role for posterior dorsolateral striatum in human habit learning. Eur. J. Neurosci. 29, 2225-32 (2009).

15. Valentin, V. V., Dickinson, A. \& O'Doherty, J. P. Determining the neural substrates of goal-directed learning in the human brain. J. Neurosci. 27, 4019-4026 (2007).

16. Watson, P., Wiers, R. W., Hommel, B. \& De Wit, S. Working for food you don't desire. Cues interfere with goal-directed food-seeking. Appetite 79, 139-148 (2014).

17. Watson, P. \& de Wit, S. Current limits of experimental research into habits and future directions. Curr. Opin. Behav. Sci. 20, 33-39 (2018).

18. Luque, D., Molinero, S., Watson, P., López, F. J. \& Le Pelley, M. E. Measuring habit

19. Lehéricy, S. et al. Distinct basal ganglia territories are engaged in early and advanced motor sequence learning. Proc. Natl. Acad. Sci. U. S. A. 102, 12566-12571 (2005). systems that encode the causal effects of actions. J. Neurosci. 28, 6750-6755 (2008).

21. Jankowski, J., Scheef, L., Hüppe, C. \& Boecker, H. Distinct striatal regions for planning and executing novel and automated movement sequences. Neuroimage 44, 
Everyday life \& Experimental Habits 1369-1379 (2009).

22. Patterson, T. K. \& Knowlton, B. J. Subregional specificity in human striatal habit learning: a meta-analytic review of the fMRI literature. Curr. Opin. Behav. Sci. 20, 7582 (2018).

23. de Wit, S. et al. Corticostriatal connectivity underlies individual differences in the balance between habitual and goal-directed action control. J. Neurosci. 32, 1206612075 (2012).

24. Brovelli, A., Nazarian, B., Meunier, M. \& Boussaoud, D. Differential roles of caudate nucleus and putamen during instrumental learning. Neuroimage 57, 1580-90 (2011).

25. Smith, K. S. \& Graybiel, A. M. Habit formation. Dialogues Clin. Neurosci. 18, 33-43 (2016).

26. de Wit, S. et al. Shifting the balance between goals and habits: Five failures in experimental habit induction. J. Exp. Psychol. Gen. 147, 1043-1065 (2018).

27. Pool, E. et al. Determining the effects of training duration on the behavioral expression of habitual control in humans: a multi-laboratory investigation. 48 (2021). doi:10.31234/OSF.IO/Z756H

28. Wechsler, K. et al. Multitasking during simulated car driving: A comparison of young and older persons. Front. Psychol. 9, 910 (2018).

29. Worringer, B. et al. Common and distinct neural correlates of dual-tasking and taskswitching: a meta-analytic review and a neuro-cognitive processing model of human multitasking. Brain Struct. Funct. 224, 1845-1869 (2019).

30. Ceceli, A. O., Myers, C. E. \& Tricomi, E. Demonstrating and disrupting well-learned habits. PLoS One 15, 1-28 (2020).

31. Cummine, J. et al. Understanding the Role of Speech Production in Reading: 
Everyday life \& Experimental Habits

Evidence for a Print-to-Speech Neural Network Using Graphical Analysis. Neuropsychology 30, 385-97 (2016).

32. Yang, Y. et al. Neural correlates of orthographic access in Mandarin Chinese writing: An fMRI study of the word-frequency effect. Front. Behav. Neurosci. 12, 1-12 (2018).

33. Lin, Z., Tam, F., Churchill, N. W., Schweizer, T. A. \& Graham, S. J. Tablet technology for writing and drawing during functional magnetic resonance imaging: A review. Sensors (Switzerland) 21, 1-22 (2021).

34. Martínez, M., Villagra, F., Castellote, J. M. \& Pastor, M. A. Kinematic and kinetic patterns related to free-walking in parkinson's disease. Sensors (Switzerland) 18, (2018).

35. Huth, A. G., De Heer, W. A., Griffiths, T. L., Theunissen, F. E. \& Gallant, J. L. Natural speech reveals the semantic maps that tile human cerebral cortex. Nature 532, 453458 (2016).

36. Martínez, M. et al. Trade-off between frequency and precision during stepping movements: Kinematic and BOLD brain activation patterns. Hum. Brain Mapp. 37, 1722-1737 (2016).

37. Oberhuber, M. et al. Functionally distinct contributions of the anterior and posterior putamen during sublexical and lexical reading. Front. Hum. Neurosci. (2013). doi:10.3389/fnhum.2013.00787

38. Varotto, S. F., Farah, H., Bogenberger, K., van Arem, B. \& Hoogendoorn, S. P. Adaptations in driver behaviour characteristics during control transitions from fullrange Adaptive Cruise Control to manual driving: an on-road study. Transp. A Transp. Sci. 16, 776-806 (2020).

39. Karimpoor, M. et al. A computerized tablet with visual feedback of hand position for 
Everyday life \& Experimental Habits

functional magnetic resonance imaging. Front. Hum. Neurosci. 9, 1-13 (2015).

40. Choi, M. H. et al. Increase in brain activation due to subtasks during driving: FMRI study using new MR-compatible driving simulator. J. Physiol. Anthropol. 36, 1-12 (2017).

41. Haynes, W. I. A. \& Haber, S. N. The organization of prefrontal-subthalamic inputs in primates provides an anatomical substrate for both functional specificity and integration: Implications for basal ganglia models and deep brain stimulation. J. Neurosci. 33, 4804-4814 (2013).

42. Eickhoff, S. B. et al. Coordinate-based activation likelihood estimation meta-analysis of neuroimaging data: A random-effects approach based on empirical estimates of spatial uncertainty. Hum. Brain Mapp. 30, 2907-2926 (2009).

43. Turkeltaub, P. E., Eden, G. F., Jones, K. M. \& Zeffiro, T. A. Meta-analysis of the functional neuroanatomy of single-word reading: Method and validation. Neuroimage 16, 765-780 (2002).

44. Lancaster, J. L. et al. Bias between MNI and talairach coordinates analyzed using the ICBM-152 brain template. Hum. Brain Mapp. 28, 1194-1205 (2007).

45. Eickhoff, S. B. et al. Behavior, sensitivity, and power of activation likelihood estimation characterized by massive empirical simulation. Neuroimage 137, 70-85 (2016).

46. Fox, P. T. et al. User manual for GingerALE 2.3. Retrieved from http://brainmap.org/ale/manual.pdf (2013).

47. Mazziotta, J. et al. A probabilistic atlas and reference system for the human brain: International Consortium for Brain Mapping (ICBM). Philos. Trans. R. Soc. Lond. B. Biol. Sci. 356, 1293-322 (2001).

48. Higashiyama, Y., Takeda, K., Someya, Y., Kuroiwa, Y. \& Tanaka, F. The neural basis 
Everyday life \& Experimental Habits

640

641

642

643

644

645

646

647

648

649

650

651

652

653

654

655

656

657

658

659

660

661

662

663

of typewriting: A functional MRI study. PLoS One 10, 1-20 (2015).

49. Gould, R. L., Brown, R. G., Owen, A. M., Ffytche, D. H. \& Howard, R. J. fMRI BOLD response to increasing task difficulty during successful paired associates learning. Neuroimage 20, 1006-1019 (2003).

50. Parkes, S. L., Bradfield, L. A. \& Balleine, B. W. Interaction of insular cortex and ventral striatum mediates the effect of incentive memory on choice between goaldirected actions. J. Neurosci. 35, 6464-6471 (2015).

51. Sommer, S. \& Pollmann, S. Putamen Activation Represents an Intrinsic Positive Prediction Error Signal for Visual Search in Repeated Configurations. Open Neuroimag. J. 10, 126-138 (2016).

52. Bellebaum, C., Koch, B., Schwarz, M. \& Daum, I. Focal basal ganglia lesions are associated with impairments in reward-based reversal learning. Brain 131, 829-841 (2008).

53. Cromwell, H. C. \& Schultz, W. Effects of expectations for different reward magnitudes on neuronal activity in primate striatum. J. Neurophysiol. 89, 2823-2838 (2003).

54. Haruno, M. \& Kawato, M. Different neural correlates of reward expectation and reward expectation error in the putamen and caudate nucleus during stimulus-action-reward association learning. J. Neurophysiol. 95, 948-959 (2006).

55. Yamada, H., Matsumoto, N. \& Kimura, M. Tonically Active Neurons in the Primate Caudate Nucleus and Putamen Differentially Encode Instructed Motivational Outcomes of Action. J. Neurosci. 24, 3500-3510 (2004).

56. Gardiner, T. W. \& Nelson, R. J. Striatal neuronal activity during the initiation and execution of hand movements made in response to visual and vibratory cues. Exp. Brain Res. 92, 15-26 (1992). 
Everyday life \& Experimental Habits

664

665

666

667

668

669

670

671

672

673

674

675

676

677

678

679

680

681

682

683

684

685

686

687

57. Wymbs, N. F., Bassett, D. S., Mucha, P. J., Porter, M. A. \& Grafton, S. T. Differential Recruitment of the Sensorimotor Putamen and Frontoparietal Cortex during Motor Chunking in Humans. Neuron 74, 936-946 (2012).

58. Cellini, N. Memory consolidation in sleep disorders. Sleep Med. Rev. 35, 101-112 (2017).

59. Andersen, K. W., Madsen, K. H. \& Siebner, H. R. Discrete finger sequences are widely represented in human striatum. Sci. Rep. 10, 1-12 (2020).

60. Romero, M. C., Bermudez, M. A., Vicente, A. F., Perez, R. \& Gonzalez, F. Activity of neurons in the caudate and putamen during a visuomotor task. Neuroreport 19, 11411145 (2008).

61. Cohen, Y., Schneidman, E. \& Paz, R. The geometry of neuronal representations during rule learning reveals complementary roles of cingulate cortex and putamen. Neuron 109, 839-851.e9 (2021).

62. Kimura, M., Kato, M. \& Shimazaki, H. Physiological properties of projection neurons in the monkey striatum to the globus pallidus. Exp. Brain Res. 82, 672-676 (1990).

63. Kimura, M., Rajkowski, J. \& Evarts, E. Tonically discharging putamen neurons exhibit set-dependent responses. Proc. Natl. Acad. Sci. U. S. A. 81, 4998-5001 (1984).

64. Kimura, M. The role of primate putamen neurons in the association of sensory stimuli with movement. Neurosci. Res. 3, 436-443 (1986).

65. Tunik, E., Houk, J. C. \& Grafton, S. T. Basal ganglia contribution to the initiation of corrective submovements. Neuroimage 47, 1757-1766 (2009).

66. Kunimatsu, J., Maeda, K. \& Hikosaka, O. The caudal part of putamen represents the historical object value information. J. Neurosci. 39, 1709-1719 (2019).

67. Peters, A. J., Fabre, J. M. J., Steinmetz, N. A., Harris, K. D. \& Carandini, M. Striatal 
Everyday life \& Experimental Habits

activity topographically reflects cortical activity. Nature 591, 420-425 (2021).

68. Haber, S. N., Fudge, J. L. \& McFarland, N. R. Striatonigrostriatal Pathways in Primates Form an Ascending Spiral from the Shell to the Dorsolateral Striatum. J. Neurosci. 20, 2369-2382 (2000).

69. Björklund, A. \& Dunnett, S. B. Dopamine neuron systems in the brain: an update. Trends Neurosci. 30, 194-202 (2007).

70. Matsuda, W. et al. Single nigrostriatal dopaminergic neurons form widely spread and highly dense axonal arborizations in the neostriatum. J. Neurosci. 29, 444-53 (2009).

71. Redgrave, P., Prescott, T. J. \& Gurney, K. The basal ganglia: a vertebrate solution to the selection problem? Neuroscience 89, 1009-1023 (1999).

72. Hikosaka, O. et al. Tutor Song Exposure Song Selectivity in the Pallial-Basal Ganglia Song Circuit of Zebra Finches Raised Without Role of the Basal Ganglia in the Control of Purposive Saccadic Eye Movements. Physiol Rev 80, 953-978 (2000).

73. Schultz, W. Behavioral theories and the neurophysiology of reward. Annu. Rev. Psychol. 57, 87-115 (2006).

74. Redgrave, P., Gurney, K. \& Reynolds, J. What is reinforced by phasic dopamine signals? Brain Research Reviews 58, 322-339 (2008).

75. Bromberg-Martin, E. S., Matsumoto, M. \& Hikosaka, O. Dopamine in Motivational Control: Rewarding, Aversive, and Alerting. Neuron 68, 815-834 (2010).

76. Ashby, F. G., Turner, B. O. \& Horvitz, J. C. Cortical and basal ganglia contributions to habit learning and automaticity. Trends in Cognitive Sciences 14, 208-215 (2010).

77. Ohbayashi, M., Picard, N. \& Strick, P. L. Inactivation of the dorsal premotor area disrupts internally generated, but not visually guided, sequential movements. J. Neurosci. 36, 1971-1976 (2016). 
Everyday life \& Experimental Habits

712

713

714

715

716

717

718

719

720

721

722

723

724

78. Nachev, P., Kennard, C. \& Husain, M. Functional role of the supplementary and presupplementary motor areas. Nature Reviews Neuroscience 9, 856-869 (2008).

79. Nambu, A., Tokuno, H. \& Takada, M. Functional significance of the corticosubthalamo-pallidal 'hyperdirect' pathway. Neurosci Res 43, 111-117 (2002).

80. Pineda-Pardo, J. A. et al. Static magnetic field stimulation of the supplementary motor area modulates resting-state activity and motor behavior. Commun. Biol. 2, 397 (2019).

81. Chen, L. L. \& Wise, S. P. Neuronal activity in the supplementary eye field during acquisition of conditional oculomotor associations. J. Neurophysiol. 73, 1101-1121 (1995).

82. Morris, N. J. \& Stein, C. M. Model-free linkage analysis of a quantitative trait. in Methods in Molecular Biology 1666, 327-342 (Humana Press Inc., 2017).

83. Haber, S. N. \& Knutson, B. The Reward Circuit: Linking Primate Anatomy and Human Imaging. Neuropsychopharmacology 35, 4-26 (2009).

84. Leisman, G., Moustafa, A. \& Shafir, T. Thinking, Walking, Talking: Integratory Motor and Cognitive Brain Function. Front. Public Heal. 4, 1 (2016).

85. Smittenaar, P., FitzGerald, T. H. B., Romei, V., Wright, N. D. \& Dolan, R. J. Disruption of Dorsolateral Prefrontal Cortex Decreases Model-Based in Favor of Model-free Control in Humans. Neuron 80, 914-919 (2013).

86. Cunnington, R., Windischberger, C., Deecke, L. \& Moser, E. The preparation and execution of self-initiated and externally-triggered movement: a study of event-related fMRI. Neuroimage 15, 373-385 (2002).

87. Aron, A. R. \& Poldrack, R. A. Cortical and Subcortical Contributions to Stop Signal Response Inhibition: Role of the Subthalamic Nucleus. J. Neurosci. 26, 2424-2433 
Everyday life \& Experimental Habits

(2006).

88. Arsalidou, M., Duerden, E. G. \& Taylor, M. J. The Centre of the Brain : Topographical Model of Motor, Cognitive , Affective , and Somatosensory Functions of the Basal Ganglia. 3054, 3031-3054 (2013).

89. McFarland, N. R. \& Haber, S. N. Thalamic relay nuclei of the basal ganglia form both reciprocal and nonreciprocal cortical connections, linking multiple frontal cortical areas. J. Neurosci. 22, 8117-8132 (2002).

90. Kim, H. F. \& Hikosaka, O. Parallel basal ganglia circuits for voluntary and automatic behaviour to reach rewards. Brain 138, 1776-1800 (2015).

91. Haber, S. N. Corticostriatal circuitry. Dialogues Clin. Neurosci. 18, 7-21 (2016).

92. Gurney, K., Humphries, M. D. \& Redgrave, P. A New Framework for Cortico-Striatal Plasticity: Behavioural Theory Meets In Vitro Data at the Reinforcement-Action Interface. PLoS Biol. 13, e1002034 (2015).

93. Alexander, G. E. \& Crutcher, M. D. Functional architecture of basal ganglia circuits: neural substrates of parallel processing. Trends in Neurosciences 13, 266-271 (1990).

94. Huang, Y., Yaple, Z. A. \& Yu, R. Goal-oriented and habitual decisions: Neural signatures of model-based and model-free learning. Neuroimage 215, 116834 (2020).

95. Liljeholm, M., Dunne, S. \& O’Doherty, J. P. Differentiating neural systems mediating the acquisition vs. expression of goal-directed and habitual behavioral control. Eur. J. Neurosci. 41, 1358-1371 (2015).

96. Eryilmaz, H. et al. Neural determinants of human goal-directed vs. habitual action control and their relation to trait motivation. Sci. Rep. 7, 1-11 (2017).

97. Peterson, B. S. et al. Human basal ganglia volume asymmetries on magnetic 
Everyday life \& Experimental Habits

resonance images. Magn. Reson. Imaging 11, 493-498 (1993).

98. Grabenhorst, F. \& Rolls, E. T. Value, pleasure and choice in the ventral prefrontal cortex. Trends Cogn. Sci. 15, 56-67 (2011).

99. Hardwick, R. M., Forrence, A. D., Krakauer, J. W. \& Haith, A. M. Time-dependent competition between goal-directed and habitual response preparation. Nat. Hum. Behav. 3, 1252-1262 (2019).

100. Wong, A. L., Goldsmith, J., Forrence, A. D., Haith, A. M. \& Krakauer, J. W. Reaction times can reflect habits rather than computations. Elife 6, (2017).

101. Amodio, D. M. The neuroscience of prejudice and stereotyping. Nat. Rev. Neurosci. 15, 670-682 (2014).

102. Vaquero, L. et al. Structural neuroplasticity in expert pianists depends on the age of musical training onset. Neuroimage 126, 106-119 (2016).

103. Cacioppo, S. et al. Intention understanding over T: A neuroimaging study on shared representations and tennis return predictions. Front. Hum. Neurosci. 8, 781 (2014).

104. Kim, W. et al. An fMRI study of differences in brain activity among elite, expert, and novice archers at the moment of optimal aiming. Cogn. Behav. Neurol. 27, 173-182 (2014).

105. Hernandez, L. F., Obeso, I., Costa, R. M., Redgrave, P. \& Obeso, J. A. Dopaminergic Vulnerability in Parkinson Disease: The Cost of Humans' Habitual Performance. Trends Neurosci. 42, 375-383 (2019).

106. Peterson, D. S. \& Horak, F. B. Neural control of walking in people with parkinsonism. Physiology 31, 95-107 (2016).

107. Wu, T. et al. Neural correlates underlying Micrographia in Parkinson's disease. Brain 139, 144-160 (2016). 
Everyday life \& Experimental Habits

784

785

786

787

788

789

790

791

792

793

794

795

796

797

108. de Wit, S., Barker, R. a, Dickinson, A. D. \& Cools, R. Habitual versus goal-directed action control in Parkinson disease. J Cogn Neurosci 23, 1218-1229 (2010).

109. Wu, T., Hallett, M. \& Chan, P. Motor automaticity in Parkinson's disease. Neurobiol. Dis. 82, 226-234 (2015).

110. Everitt, B. J. et al. Neural mechanisms underlying the vulnerability to develop compulsive drug-seeking habits and addiction. Philos. Trans. R. Soc. B Biol. Sci. 363, 3125-3135 (2008).

111. Neal, D. T., Wood, W. \& Quinn, J. M. Habits: A Repeat Performance. Curr. Dir. Psychol. Sci. 15, 198-202 (2006).

112. Lally, P., van Jaarsveld, C. H. M., Potts, H. W. W. \& Wardle, J. How are habits formed: Modelling habit formation in the real world. Eur. J. Soc. Psychol. 40, 9981009 (2010). 
Everyday life \& Experimental Habits

798

799

800

801

802

803

804

805

806

807

808

809

810

811

812

813

814

815

816

817

818

819

820

821

822

823

824

825

826

827

828

829

830

\section{Acknowledgments}

We are thankful to Blanca Caijao and Cristina Rubio (Universidad Rey Juan Carlos)

for their help in article search. MM is funded by a PhD Fellowship from Instituto de Salud

Carlos III (PFIS-FI720/00068). IO is funded by Instituto de Salud Carlos III (Miguel Servet,

CP18/00038) and AES-ISCIII (PI19/00298) from Ministry of Science and Innovation in Spain.

The funders played no role in the idea, design, data collection or analysis, decision to publish or manuscript editing and writing.

\section{Author Contributions}

P.G. design, data collection, writing; M.M. design, data collection, analysis, writing;

P.R. writing; D.L. design, data collection, writing; I.O. design, data collection, writing;

\section{Competing Interests statement}

The authors declare no competing interest.

Figure Legends and Tables

Figure 1. Hypothesis diagram on striatal role in both everyday-life and experimental habits. Activities part of daily life such as writing, reading, walking or driving were selected as everyday-life habits (see Box 1 for task measurements and details) to expect a critical striatal role in executing these habits forms. Similar striatal activities can be expected compared to experimental paradigms commonly used in the cognitive science literature.

Figure 2. Everyday-life and experimental thresholded activation maps. a) Axial views for the main regions. Overlap regions at the right column are shown in blue. Note that there exists activation in the cerebellar cortex for the case of everyday-life studies but it is omitted here for brevity. $Z=52$ view for the experimental studies is shown as an unthresholded map for visualization purposes (ALE value $\approx 0.01$ ) (b) $3 \mathrm{D}$ striatum reconstruction showing all the activation that fall inside it. Overlap regions at the right column are shown in blue. c) 3D striatum reconstruction showing the differential activation of the Everyday -life > Experimental contrast (in green) and the Experimental > Everyday-life contrast (in red).

Figure 3. Everyday-life cognitive-motor subcategories thresholded activation maps. a) Axial views for the main regions. Overlap regions at the right column are shown in blue. b) 3D striatum reconstruction showing all the activation that fall inside it. Overlap regions at the right column are shown in blue. c) Foci distribution of all the studies that fall in the striatum. Note that some foci may correspond to the same study.

Figure 4. Experimental subcategories thresholded activation maps. a) Axial views for the main regions. In this case there are no overlap regions at the right column. b) 3D striatum 
bioRxiv preprint doi: https://doi.org/10.1101/2021.07.02.450904; this version posted July 4, 2021. The copyright holder for this preprint (which was not certified by peer review) is the author/funder. All rights reserved. No reuse allowed without permission.

Everyday life \& Experimental Habits

831 reconstruction showing all the activation that fall inside it. In this case there are no overlap regions at the right column. c) Foci distribution of all the studies that fall in the striatum. Note 833 that some foci may correspond to the same study. 
Table 1. Everyday-life habits peaks coordinates in MNI152 space with region names from Harvard-Oxford atlas. Percentages of each brain region indicate how much activation from a cluster fall into such region. Coordinates of any activation comprising < $5 \%$ of its volume in a region are not shown for conciseness.

\begin{tabular}{|c|c|c|c|c|c|c|}
\hline \multirow{2}{*}{$\begin{array}{l}\text { Cluster } \\
\text { ID }\end{array}$} & \multirow{2}{*}{$\begin{array}{c}\text { Volume } \\
\left(\mathrm{mm}^{3}\right)\end{array}$} & \multirow{2}{*}{ Brain regions $(\%)$} & \multicolumn{3}{|c|}{$\begin{array}{c}\text { MNI peak } \\
\text { coordinates }\end{array}$} & \multirow[t]{2}{*}{ ALE value } \\
\hline & & & $\mathbf{x}$ & $\mathbf{y}$ & $\mathbf{z}$ & \\
\hline \multirow{3}{*}{1} & \multirow{3}{*}{8792} & Left Putamen (53.14\%) & -24 & -4 & 6 & 0.074 \\
\hline & & Left Thalamus (28.84\%) & -14 & -22 & 10 & 0.04 \\
\hline & & Left Pallidum (15.74\%) & -23 & -4 & 2 & 0.054 \\
\hline \multirow{4}{*}{2} & \multirow{4}{*}{5320} & Right Putamen (53.38\%) & 24 & 4 & 6 & 0.054 \\
\hline & & Right Thalamus (20.45\%) & 16 & -16 & 8 & 0.035 \\
\hline & & Right Insula (13.08\%) & 32 & 14 & 8 & 0.037 \\
\hline & & Right Pallidum (9.47\%) & 20 & 2 & 5 & 0.037 \\
\hline \multirow{4}{*}{3} & \multirow{4}{*}{4840} & Left Supplementary Motor Area (42.64\%) & -6 & 0 & 52 & 0.035 \\
\hline & & Right Paracingulate Gyrus (15.87\%) & 6 & 14 & 50 & 0.031 \\
\hline & & Right Supplementary Motor Area (15.37\%) & 4 & 8 & 56 & 0.03 \\
\hline & & Left Paracingulate Gyrus (13.06\%) & 0 & 8 & 53 & 0.027 \\
\hline 4 & 3232 & Left Precentral Gyrus (97.03\%) & -54 & -2 & 40 & 0.038 \\
\hline 5 & 2416 & Right-Cerebellum-Cortex (100\%) & 8 & -64 & -20 & 0.037 \\
\hline \multirow{4}{*}{6} & \multirow{4}{*}{2368} & Left Central Opercular Cortex (53.38\%) & -46 & -2 & 6 & 0.037 \\
\hline & & Left Precentral Gyrus (18.58\%) & -56 & 8 & 4 & 0.033 \\
\hline & & Left Inferior Frontal Gyrus pars opercularis $(18.58 \%)$ & -56 & 10 & 24 & 0.024 \\
\hline & & Left Insula (7.09\%) & -44 & -1 & 4 & 0.03 \\
\hline \multirow{3}{*}{7} & \multirow{3}{*}{2208} & Right Precentral Gyrus (76.09\%) & 56 & 12 & 32 & 0.041 \\
\hline & & Right Inferior Frontal Gyrus pars opercularis $(18.48 \%)$ & 54 & 13 & 31 & 0.036 \\
\hline & & Right Middle Frontal Gyrus (5.43\%) & 46 & 13 & 30 & 0.013 \\
\hline \multirow{5}{*}{8} & \multirow{5}{*}{1152} & Right Inferior Frontal Gyrus pars opercularis (34.03\%) & 54 & 10 & 0 & 0.035 \\
\hline & & Right Central Opercular Cortex (27.08\%) & 52 & 6 & 2 & 0.029 \\
\hline & & Right Temporal Pole (13.89\%) & 54 & 10 & -2 & 0.034 \\
\hline & & Right Precentral Gyrus (11.81\%) & -3 & -14 & 54 & 0.022 \\
\hline & & Right Planum Polare (9.03\%) & 53 & 5 & -2 & 0.028 \\
\hline \multirow{2}{*}{9} & \multirow{2}{*}{1032} & Left Precentral Gyrus (42.64\%) & -26 & -8 & 56 & 0.04 \\
\hline & & Left Superior Frontal Gyrus (36.43\%) & 6 & 11 & 54 & 0.029 \\
\hline
\end{tabular}


Table 2. Experimental habits peaks coordinates in MNI152 space with region names from Harvard-Oxford atlas. Percentages of each brain region indicate how much activation from a cluster fall into such region. Coordinates of any activation comprising < $5 \%$ of its volume in a region are not shown for conciseness.

\begin{tabular}{|c|c|c|c|c|c|c|}
\hline \multirow{2}{*}{$\begin{array}{c}\text { Cluster } \\
\text { ID }\end{array}$} & \multirow{2}{*}{$\begin{array}{c}\text { Volume } \\
\left(\mathrm{mm}^{3}\right)\end{array}$} & \multirow{2}{*}{ Brain regions (\%) } & \multicolumn{3}{|c|}{ MNI peak coordinates } & \multirow{2}{*}{ ALE value } \\
\hline & & & $\mathbf{x}$ & $\mathbf{y}$ & $\mathbf{z}$ & \\
\hline \multirow{5}{*}{1} & \multirow{5}{*}{3344} & Right Caudate (44.74\%) & 12 & 6 & 10 & 0.028 \\
\hline & & Right Pallidum (20.57\%) & -14 & 5 & -6 & 0.025 \\
\hline & & Right Accumbens (15.31\%) & 10 & 8 & -6 & 0.03 \\
\hline & & Right Putamen (10.05\%) & 14 & 9 & -6 & 0.025 \\
\hline & & Right Thalamus $(6.46 \%)$ & 11 & -1 & 11 & 0.02 \\
\hline \multirow{4}{*}{2} & \multirow{4}{*}{2088} & Left Putamen (30.27\%) & -14 & 5 & -9 & 0.029 \\
\hline & & Left Accumbens (27.97\%) & -12 & 6 & -10 & 0.033 \\
\hline & & Left Caudate (20.69\%) & -10 & 4 & 4 & 0.017 \\
\hline & & Left Pallidum (12.26\%) & 14 & 5 & -6 & 0.025 \\
\hline 3 & 776 & Left Putamen (98.97\%) & -30 & -8 & -2 & 0.02 \\
\hline \multirow{2}{*}{4} & \multirow{2}{*}{744} & Right Insula (90.32\%) & 32 & 22 & -4 & 0.023 \\
\hline & & Right Orbito-Frontal Cortex (9.68\%) & 29 & 22 & -7 & 0.019 \\
\hline
\end{tabular}


Box 1. Description of most used paradigms to assess everyday-life (A) behaviours and experimental learning $(B)$ in $\mathrm{fMRI}$ contexts.

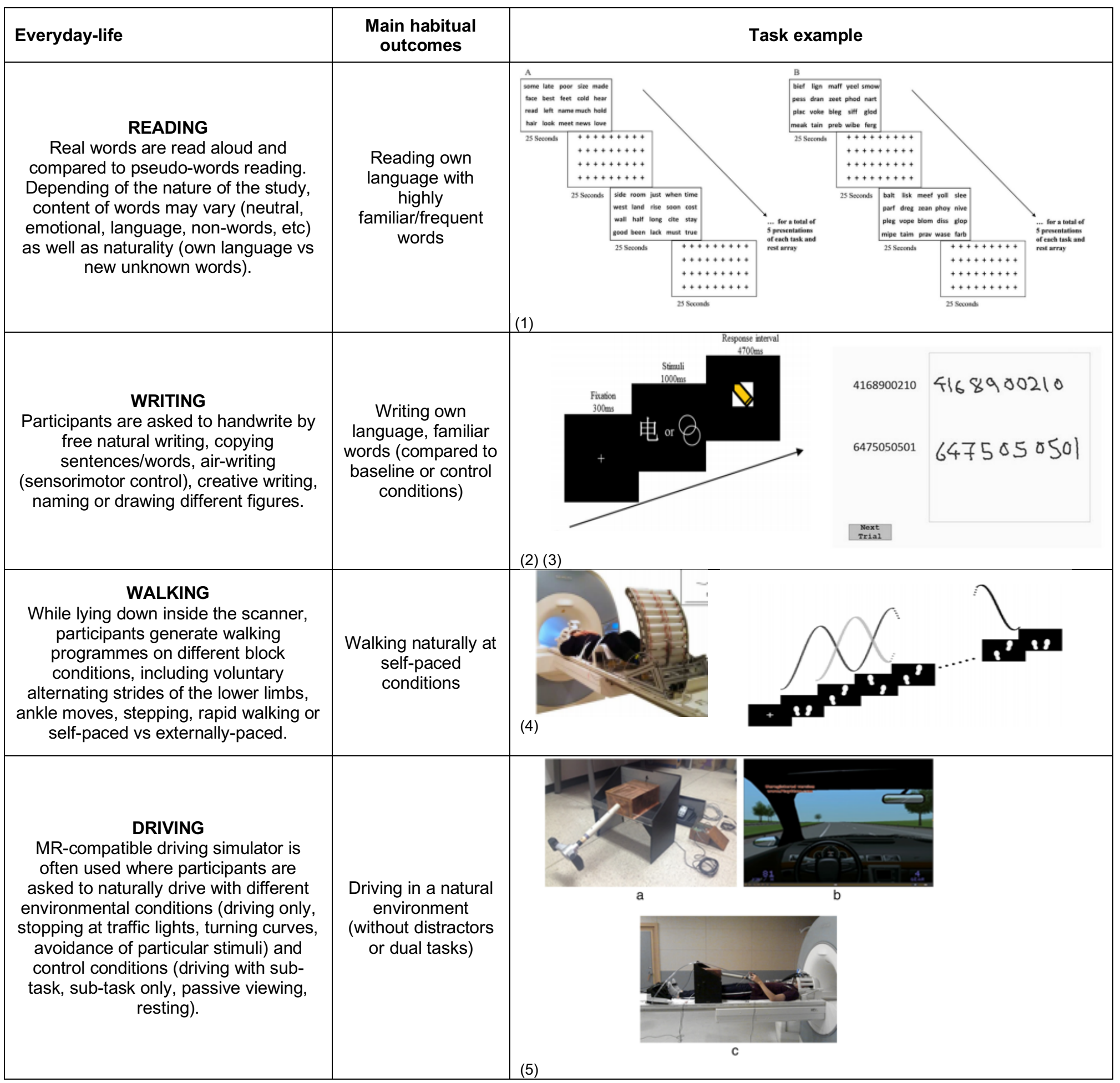

(1) Cummine et al., 2016; (2) Yang et al., 2018; (3) Karimpoor et al., 2015; (4) Martínez et al., 2016; (5) Choi et al., 2017. 


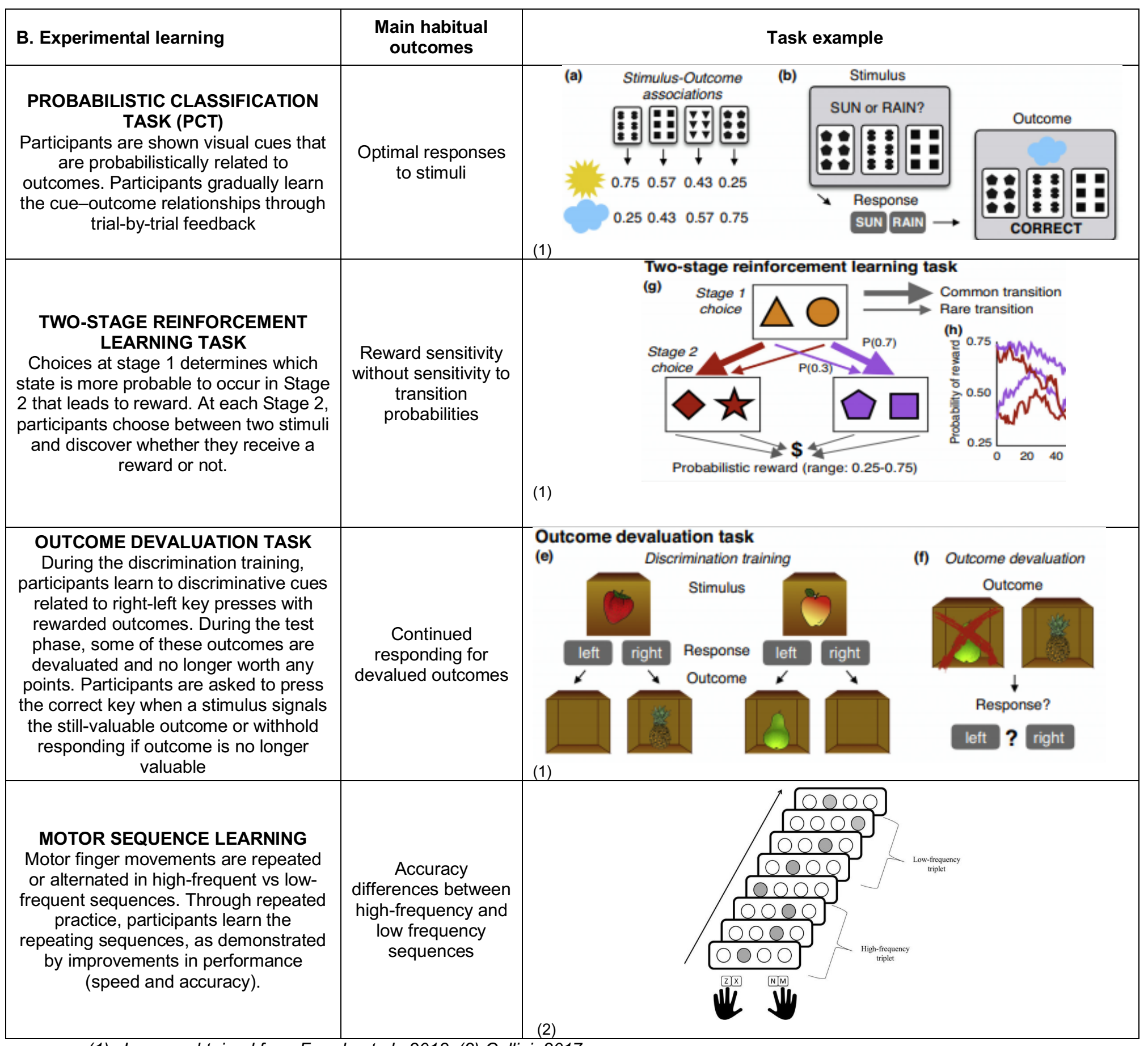

(1) Images obtained from Foerde et al., 2018; (2) Cellini, 2017 


\section{Supplementary material}

\section{Striatal role in everyday-life and laboratory-developed habits}

Pasqualina Guida ${ }^{1,2,6 *}$, Mario Michiels ${ }^{1,2,6 *}$, Peter Redgrave ${ }^{5}$, David Luque ${ }^{3,4}$, Ignacio Obeso $^{1,2}$

${ }^{*}$ Co-first authors

${ }^{1}$ CINAC, Hospital Universitario HM Puerta del Sur, Móstoles, Madrid, Spain ${ }^{2}$ CIBERNED, Instituto de Salud Carlos III, Madrid, Spain

${ }^{3}$ Departamento de Psicología Básica, Universidad Autónoma de Madrid, Madrid, Spain

${ }^{4}$ Departamento de Psicología Básica, Universidad de Málaga, Madrid, Spain

${ }^{5}$ Department of Psychology, University of Sheffield, Sheffield, S10 2TN, UK

${ }^{6} \mathrm{PhD}$ program in Neuroscience, Universidad Autónoma de Madrid, 28029 Madrid, Spain

\section{Methods}

The complete set of search terms were as follows:

(1) Everyday life:

(walking[MeSH Terms] OR walking[Title/Abstract]) OR (Driving behaviour[MeSH Terms] OR Driving behaviour[Title/Abstract]) OR (Driving behavior[MeSH Terms] OR Driving behavior[Title/Abstract]) OR (car driving[MeSH Terms] OR Car driving[Title/Abstract]) OR (writing[MeSH Terms] OR writing[Title/Abstract]) OR (handwriting[MeSH Terms] OR handwriting[Title/Abstract]) OR (reading[Title/Abstract] OR reading[MeSH Terms) AND (functional magnetic resonance imaging[MeSH Terms] OR fMRI[Title/Abstract]) AND ((basal ganglia[Title/Abstract] OR caudate[Title/Abstract] 
OR putamen[Title/Abstract] OR striatum[Title/Abstract]) OR (basal ganglia[MeSH Terms] OR caudate nucleus[MeSH Terms] OR putamen[MeSH Terms] OR striatum[MeSH Terms]))

(2) Experimental:

(“habit" OR "habits" OR "probabilistic classification" OR "weather prediction" OR "response learning” OR “instrumental conditioning" OR “instrumental learning" OR "reinforcement learning" OR "outcome devaluation" OR "sequential decision" OR "two step” OR “2 step”) AND (“basal ganglia” OR “caudate” OR "putamen” OR "striatum”) AND ("fMRI" OR "functional magnetic resonance imaging" OR "functional MRI") AND ("2017/06/22"[PDAT] : "3000/12/31"[PDAT]) 
Table S1. Studies with everyday-life habits included in the meta-analysis.

\begin{tabular}{|c|c|c|c|c|c|}
\hline Study by domain & $n=1441$ & Foci & Task & Contrast & Statistical threshold \\
\hline \multicolumn{6}{|l|}{ Writing } \\
\hline Katanoda et al. (2001) & 17 & 19 & $\begin{array}{l}\text { Write a name of an object or } \\
\text { naming the object }\end{array}$ & Writing $>$ naming & $\begin{array}{l}p<0.001 \text { voxel-level \& } p<0.05 \\
\text { cluster-level corrected }\end{array}$ \\
\hline Nakamura et al. (2002) & 9 & 10 & $\begin{array}{l}\text { Upon auditory presentation } \\
\text { subjects wrote kanji word. }\end{array}$ & Writing $>$ Rest & $\begin{array}{l}p<0.05 \text { corrected for multiple } \\
\text { comparison }\end{array}$ \\
\hline Beeson et al. (2003) & 12 & 29 & $\begin{array}{l}\text { Writing words or drawing } \\
\text { circles }\end{array}$ & Writing words $>$ drawing circles & $p<0.001$ uncorrected \\
\hline Hu Xing-yue et al. (2006) & 10 & 11 & Writing with a pencil & Main Effect of Writing with a pencil & $p<0.0001$ uncorrected \\
\hline Segal et al. (2011) & 9 & 19 & $\begin{array}{l}\text { Writing or naming pictures of } \\
\text { objects and drawing one loop } \\
\text { per syllable of the object's } \\
\text { name }\end{array}$ & Writing $>$ naming plus loops & $p<0.05$ corrected \\
\hline Horovitz et al. (2013) & 13 & 5 & $\begin{array}{l}\text { Writing, tapping, and } \\
\text { zigzagging with each limb }\end{array}$ & Right-handwriting > other tasks & $p<0.001$ FWE corrected \\
\hline Erhard et al. (2014) & 20 & 13 & Creative writing & $\begin{array}{l}\text { Main effects of creative writing in } \\
\text { expert writers }\end{array}$ & $p<0.05$ FWE corrected \\
\hline Longcamp et al. (2014) & 18 & 13 & Writing of letters or digits & Writing $>$ holding the pen still & $\begin{array}{l}p<0.05 \text { voxel-level FWE } \\
\text { corrected }\end{array}$ \\
\hline Potgieser et al. (2015) & 16 & 21 & $\begin{array}{l}\text { Write a sentence or hand } \\
\text { tapping }\end{array}$ & $\begin{array}{l}\text { Right-handwriting > right-hand } \\
\text { tapping }\end{array}$ & $\begin{array}{l}p<0.001 \text { voxel-level } \& p<0.05 \\
\text { cluster-level FWE corrected } \& k \geq 8\end{array}$ \\
\hline Bisio et al. (2017) & 7 & 25 & Writing sentences & Writing > resting & $p<0.05$ FWE corrected \\
\hline Karimpoor et al. (2018) & 12 & 38 & $\begin{array}{l}\text { Copying grocery lists, phone } \\
\text { numbers or sentences }\end{array}$ & All handwriting tasks $>$ resting & $p<0.05$ FDR corrected \\
\hline Yang et al. (2018) & 34 & 29 & Copying chinese characters & $\begin{array}{l}\text { Writing high frequency > writing low } \\
\text { frequency }\end{array}$ & $p<0.05$ FDR corrected \\
\hline \multicolumn{6}{|l|}{ Reading } \\
\hline Bookheimer et al. (1995) & ) 16 & 33 & $\begin{array}{l}\text { Reading words or naming } \\
\text { objects }\end{array}$ & Main effect of read aloud & $p<0.001$ corrected \\
\hline Moore et al. (1999) & 8 & 7 & Reading/naming & Main effect of reading words & $p<0.001$ uncorrected \\
\hline Mechelli et al. (2003) & 20 & 10 & Early/late reading processing & Early reading $>$ Fixation & $p<0.05$ corrected \\
\hline Buchsbaum et al. (2005) & 17 & 15 & Reading/Hearing & Reading $>$ Control & $\begin{array}{l}z>2.33 \& p<0.01 \text { cluster- } \\
\text { corrected }\end{array}$ \\
\hline Vigneau et al. (2005) & 23 & 39 & $\begin{array}{l}\text { Reading words/ non reading } \\
\text { letters }\end{array}$ & Read $>$ Cross fixation & $p<0.001$ uncorrected \\
\hline Meschyan et al. (2006) & 12 & 12 & Reading different languages & Reading $>$ Resting & $\begin{array}{l}p<0.001 \text { uncorrected \& } p<0.05 \\
\text { corrected spatial extent threshold }\end{array}$ \\
\hline Binder et al. (2006) & 30 & 31 & Non-word reading & Reading > Fixation & $\begin{array}{l}p<0.00001 \text { uncorrected } \& p< \\
0.05 \text { corrected }\end{array}$ \\
\hline Carreiras et al. (2007) & 36 & 32 & Reading/Lexical decision & Reading > Baseline & $p<0.05$ corrected \\
\hline Church et al. (2008) & 50 & 37 & Reading/Repeat & Reading $>$ Repeat & $\begin{array}{l}p<0.05 \text { corrected with } k \geq 24 \\
\text { voxels }\end{array}$ \\
\hline Yarkoni et al. (2008) & 28 & 21 & $\begin{array}{l}\text { Reading/Comprehension/lexic } \\
\text { al decision }\end{array}$ & Reading > Fixation & $p<0.05$ uncorrected \\
\hline Seghier et al. (2008) & 43 & 5 & Reading aloud/fixation & Reading > Fixation & $p<0.05$ corrected \\
\hline Seghier et al. (2010) & 28 & 5 & Reading aloud/naming & Reading > Fixation & $p<0.05$ corrected \\
\hline Oberhuber et al. (2013) & 25 & 6 & Reading/naming & Reading $>$ Naming & $\begin{array}{l}p<0.05 \text {, cluster level FWE- } \\
\text { corrected }\end{array}$ \\
\hline Vannest et al. (2013) & 49 & 11 & Reading/Generate words & Reading $>$ Generate words & $\begin{array}{l}p \leq 0.01 \text { voxel-level corrected } \& \mathrm{t} \\
\geq 7.5, \text { cluster size } 30\end{array}$ \\
\hline
\end{tabular}


Hsu et al. (2015)

Rueckl et al. (2015)

Cummine et al. (2016)

Oberhuber et al. (2016) 26
24

$84 \quad 18$

$15 \quad 19$

22

193
Reading emotional or neutral sentences

Cheema et al. (2018)
Reading/Hearing

Reading words/pseudowords

Reading words/ pseudowords or naming object/ colors

Reading words / non words
Reading $>$ Resting

Reading $>$ Resting

Main effect of reading real words

Reading words $>$ other conditions

Reading $>$ Control $p<0.005$ voxel-level $\& p<0.05$

cluster-level FDR corrected

$p<0.001$ FDR corrected

$p<0.001$ uncorrected

$p<0.05$ FWE corrected

$p<0.0001$ uncorrected

Walking

Ciccarelliet al. (2005) $\quad 16 \quad 9 \quad \begin{aligned} & \text { Right-left foot passive } \\ & \text { movement }\end{aligned}$
movement

Externally generated

Christensen et al. (2007) 18

10

movement with or without visual feedback

la Fougère et al. (2010) 16

Trinastic et al (2010) 8

Swinnen et al. (2010)

14

Toyomura et al. (2012)

12

Sauvagea et al. (2013)

Martinez et al. (2014)

Lukas Jaeger et al.

(2014)

Noble et al. (2014)

Martín et al. (2016)

Marchal et al. (2019)

Peters et al. (2019)

Allali G et al. (2019)

\section{Driving}

Uchiyama et al. (2003)

\section{2}

19

$20 \quad 24$ Active stepping

14

Real locomotion

Active ankle dorsiflexion

$90^{\circ}$ out-of-phase versus iso task

9 Self-paced condition

\section{Speed execution task}

20 Stepping condition

Bilateral plantarflexion

\section{exertion against physical}

resistance

1916 Pseudo-gait

$\begin{array}{lll}20 & 7 & \text { Virtual gait task with doorway }\end{array}$

$2213 \quad$ Ankle Task

$326 \quad 6 \quad$ Varying walking speeds
Passive > Active movement

$p<0.05$ corrected

Conjunctions of externally generated movements (regardless of feedback)

Walking $>$ Resting condition

$p<0.05$ FDR corrected

Ankle dorsiflexion > Plantarflexion

$p<0.05$

$90^{\circ}$ Left $>$ Iso directional Left

Self $>$ Externally paced

Fast execution $>$ low execution

Main effect of walking

Main effect active walking

Bilateral > Unilateral

$\mathrm{p}<0.001$ FDR corrected

$p<0.0001 \& k \geq 10$ uncorrected; $p$

$<0.05$ FWE corrected

$p<0.05$ corrected

$p<0.001$ FDR corrected

$p<0.001$ cluster-level corrected \& $k \geq 42$ voxels

$p<0.01$ FWE corrected

Main effect of stability of stepping frequencies

Gait doorway > Walkway doorway

Ankle main effect

Rapid walking $>$ Normal walking speed $p<0.05$ FWE corrected

$\mathrm{p}<0.001$ uncorrected

$p<0.005$ uncorrected

$p<0.05$ cluster-level corrected
Graydon et al. (2004) $670 \quad$ Driving simulator task

Spiers et al. (2007)

Callan et al. (2009)

Hsieh et al. (2009)

Chein et al. (2011)

Uchiyama et al. (2012)

18

17

16

208 The getaway game

$14 \quad 18$ In-car video assist system

2823 Static Load Paradigm

402 Stoplight driving game

Driving simulator task (with dual task conditions)

Chung et al. (2014)

\section{Driving simulator task}

Active $>$ Passive driving

Simulated driving $>$ Fixation

Turning $L>$ Turning $R$

Driver's perspective with truck blocking viewing $>$ driver's perspective occluded with a video from the perspective of the camera

Driving video no distractor $>$ fixation

Drive peer $>$ alone

Driving main effect

Driving only $>$ Driving with task $p<0.05$ cluster-level FWE corrected

$p<0.05$ corrected

$p<0.001$ uncorrected \& $k \geq 5$

$p<0.05$ FDR corrected

$$
p<0.0001 \text { corrected }
$$

$p<0.05$ FWE corrected $p<0.001$ voxel level \& $p<0.05$ cluster level corrected

$p<0.05$ corrected 
Table S2. Studies with experimental habits included in the meta-analysis.

\begin{tabular}{|c|c|c|c|c|c|}
\hline Study by domain & $n=973$ & Foci & Task & Contrast (short) & Statistical threshold \\
\hline
\end{tabular}

\section{Probabilistic learning}

Poldrack et al. (1999)

Poldrack et al. (2001)

Aron et al. (2004)

Delgado et al. (2005)

Fera et al. (2005)

Aron et al. (2006)

Foerde et al. (2006)

14

Glascher et al. (2010)

18

Celone et al. (2011)

19

Wunderlich et al. (2012)

Soares et al. (2012)

Schwabe et al. (2013)

Lee et al. (2014)

Deserno et al. (2015)

Doll et al. (2015)

Dunne et al. (2016)

Oh-Descher et al. (2017)

Nebe et al. (2017) 146 35

2-step probabilistic learning task

Erdeniz et al. (2019)

19

Mas-Herrero et al. (2019) 20

15

Probabilistic learning task

Probabilistic learning task 15

17

18

5

Probabilistic learning task

8 Probabilistic learning task 75

$22 \quad 5 \quad 2$-step probabilistic learning $29 \quad 7$

$20 \quad 2$ 2-step probabilistic learning

173 Probabilistic learning task

25

3

Probabilistic learning task

Others

Gottfried et al. (2003)

laria et al. (2003)

Lehéricy et al. (2005)

Forstmann et al. (2008)

13

7

Associative learning

$7 \quad 5$

5 Maze navigation

143 Motor sequence learning

$193 \quad$ Moving dots task Task > control (perceptual-motor) $\begin{aligned} & \mathrm{p}<0.05 \text { corrected with Gaussian } \\ & \text { Random Field Theory }\end{aligned}$

Task $>$ control (perceptual-motor) $p<0.005$ uncorrected $\& k \geq 5$ voxels

Task $>$ baseline

$p<0.05$ FDR corrected

Condition and time interaction

$p<0.0001$ uncorrected $\& k \geq 5$ voxels

Task $>$ control (perceptual-motor) $p<0.05$ uncorrected

$z>2.3$ voxel-level \& $p<0.01$ cluster-

Task $>$ control (perceptual-motor) level corrected with Gaussian Random Field Theory

Modulation by performance (accuracy)

$p<0.05$ corrected with Gaussian Random Field Theory

RPE

$p<0.006$ corrected \& $k \geq 10$

Task > control (perceptual-motor)

$p<0.01$ voxel-level \& $p<0.05$ cluster-level corrected

Trained $>$ Planning (RPE)

$p<0.05$ FWE corrected \& $k \geq 5$

Stress $>$ control

$p<0.05$ FWE corrected

Task $>$ control (perceptual-motor) $p<0.05$ FWE corrected $\& k \geq 5$

RPE

$p<0.05$ FWE corrected

Model-free component

$p<0.05$ corrected

RPE

$p \leq 0.0005$ cluster-level FWE corrected

RPE

High time pressure > low time pressure (Positive subjective sum of evidence)

$p<0.05$ corrected

$p<0.005$ voxel-level $\& p<0.05$ cluster-level corrected

RPE

$p<0.05$ FWE corrected

CS familiar > CS novel

$p<0.005$ uncorrected

RPE (pseudofeedback)

$\mathrm{p}<0.001$ corrected
Outcome devaluation

$p<0.001$ uncorrected

Task > control (perceptual-motor)

Modulation by task experience

Speed $>$ accuracy $p<0.05$ corrected

$p<0.0001$ voxel-level $\& p<0.05$ cluster-level corrected

$p<0.001$ corrected 
Fernández-Seara et al. (2009)

Tricomi et al. (2009)

Worthy et al. (2010)

Steele et al. (2010)

Brovelli et al. (2011)

Beierholm et al. (2011)

De Wit et al. (2012)

Etchamendy et al. (2012) 15

Liljeholm et al. (2012)

$$
15
$$

Liljeholm et al. (2015)

19

4 Sequential choice

Fermin et al. (2016)

$$
18
$$

13

Sequential choice

Eryilmaz et al. (2017)

72

9

Grid-Sailing

Van Steenbergen et al. (2017)

5

Discriminative learning

Zwosta et al. (2018)

53

\section{5}

Discriminative learning

Watson et al. (2018)

29

Discriminative learning

Anggraini et al. (2018)
16 Maze navigation
Late > early

Late > early

State-change uncertainty

Late > early

$\mathrm{P}$ (correct | past observations) $>\mathrm{P} P<0.05$ FWE corrected (correct by chance)

Model-free component

$p<0.001$ uncorrected $\& k \geq 5$

Negative predictors of goaldirected action (outcome

$p<0.005$ uncorrected $\& k \geq 20$ devaluation)

Q-signal, learners

$p<0.05$ corrected

Decreased activity in the R-O but $p<0.05$ corrected with cluster size not int the S-R thresholding

Modulation by devaluation insensitivity

Previous S-R learning - Modelbased conditions

Incongruent > standard cue

Cue-driven $>$ Value-driven action control

Decrease reward and no reward (Contingency degradation)

$p<0.05$ cluster-level corrected

$p<0.0001$ uncorrected

$p<0.001$ voxel-level $\& p<0.05$ cluster-level corrected

$p<0.001$ uncorrected $\& k \geq 15$

Slip of action trials $>$ still-valued responses

Model-free component $p<0.001$ voxel-level $\& p<0.05$ cluster-level corrected

$p<0.05$ corrected

$p<0.001$ voxel-level $\& p<0.05$ cluster-level corrected 
bioRxiv preprint doi: https://doi.org/10.1101/2021.07.02.450904; this version posted July 4, 2021. The copyright holder for this preprint (which was not certified by peer review) is the author/funder. All rights reserved. No reuse allowed without permission.

Figure S1. PRISMA chart describing the steps followed in the selection of studies.

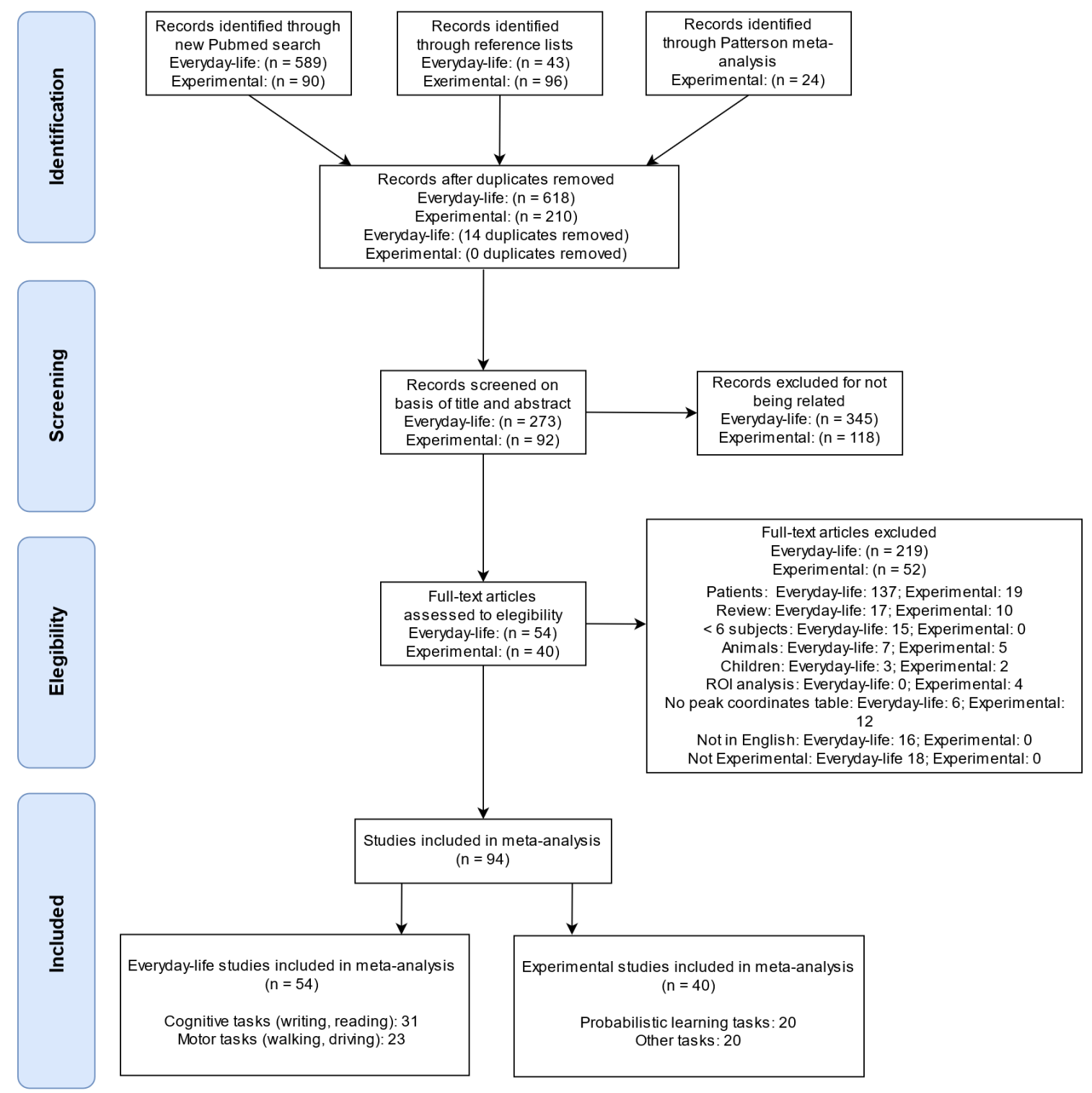

